Recepción: 20 / 04 / 2017

Aceptación: 20 / 05 / 2017

Publicación: 15 / 09 / 2017

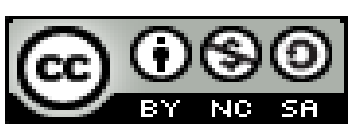

Ciencia de la computación

Artículo Científico

\title{
Guía de estilo para sistemas eléctricos de potencia
}

\section{Style guide for electric power systems}

\section{Guia de estilo para sistemas de energia elétrica}

Raul C. Ulloa De Souza I

raululloa88@gmail.com

\begin{abstract}
Alejandro J. Martinez-Peralta II
pipoperalta1990@hotmail.com

Maria E. Moreno-Sanchez ${ }^{\text {III }}$

maria-moreno1@gamil.com

Byron F. Chere-Quiñonez IV

cherokyfernando@hotmail.com
\end{abstract}

Correspondencia: raululloa88@gmail.com

I Docente de la Universidad Técnica Luis Vargas Torres de Esmeraldas, Esmeraldas, Ecuador.

II Docente de la Universidad Técnica Luis Vargas Torres de Esmeraldas, Esmeraldas, Ecuador.

III Docente de la Universidad Técnica Luis Vargas Torres de Esmeraldas, Esmeraldas, Ecuador.

IV Docente de la Universidad Técnica Luis Vargas Torres de Esmeraldas, Esmeraldas, Ecuador. 


\section{Resumen}

En el presente trabajo se diseña una guía de estilo para la identificación de los elementos de un sistema eléctrico de potencia, cuyos parámetros son almacenados en una base de datos creada en Microsoft Acces y que pueden ser leídos a través de la función "CargarTablaBases” la misma que pertenece al código de programación planteado.

Así mismo permite el ingreso mediante tablas de Excel del estado de los interruptores y seccionadores propios del sistema a modelarse. Para este caso se usa configuración de las subestaciones "San Nicolás”, "Las Peñas”, “Álvaro Enríquez”, "Cuatro Colinas”, "Los Laureles I” y “Los Laureles II" pertenecientes al Sistema Nacional de Transmisión, todas subestaciones de llegada de subtransmisión con niveles de voltaje de $46 \mathrm{kV} / 23 \mathrm{kV}$ y $46 \mathrm{kV} / 13.8 \mathrm{kV}$ respectivamente.

Se presenta un código de programación, utilizando el software Matlab, que permita evaluar el estado de conexión de los elementos de dichas subestaciones, principalmente transformadores, líneas de transmisión que conforman el anillo y el estado final de los interruptores y seccionadores.

El programa planteado en el presente Proyecto arroja como resultados todos los transformadores en servicio, así como las líneas de transmisión excepto la que une las subestaciones "Los Laureles I" y "Los Laureles II". Estos resultados se sujetan al estado de los interruptores ingresado, es decir que al cambiar dicha matriz se obtienen resultados distintos, esto se debe a que el programa es general, es decir que se puede analizar el estado de cualquier sistema eléctrico de potencia cuyos parámetros hayan sido ingresados previamente a través de la base de datos en Acces y el estado de los interruptores a través de Excel.

Palabras claves: CargarTablaBases; Excel; Acces. 


\section{Abstract}

In the present work, a style guide is designed for the identification of the elements of an electrical power system, whose parameters are stored in a database created in Microsoft Access and that can be read through the function "LoadBaseBases" same that belongs to the programming code raised.

Also allows the entry through Excel tables of the state of the switches and disconnectors of the system to be modeled. For this case the configuration of the substations "San Nicolás", "Las Peñas", "Álvaro Enríquez", "Cuatro Colinas", "Los Laureles I" and "Los Laureles II" belonging to the National Transmission System are used, all substations arrival of subtransmission with voltage levels of $46 \mathrm{kV} / 23 \mathrm{kV}$ and $46 \mathrm{kV} / 13.8 \mathrm{kV}$ respectively.

A programming code is presented, using Matlab software, which allows to evaluate the state of connection of the elements of these substations, mainly transformers, transmission lines that make up the ring and the final state of the switches and disconnectors.

The program proposed in this project results in all the transformers in service, as well as the transmission lines except the one that connects the substations "Los Laureles I" and "Los Laureles II". These results are subject to the state of the switches entered, ie that when changing this matrix different results are obtained, this is because the program is general, ie it is possible to analyze the state of any electrical power system whose parameters have been entered previously through the database in Acces and the state of the switches through Excel.

Key words: LoadTableBases; Excel; Access. 


\section{Introducción.}

Las guías de estilo en Sistemas de Generación, Transmisión o Distribución, son de suma importancia, ya que permiten identificar cada uno de los elementos que componen el sistema en general, así mismo sirven como base para la codificación de estos, lo que es fundamental para la utilización de la herramienta computacional, pilar de los sistemas de Suministro de Energía Eléctrica (SSEE), ya sea en el área información que permiten llevar un control georeferenciado y automatizado del sistema en general. [1-3]

En el presente proyecto se plantea la nomenclatura a ser utilizada en cada uno de los elementos que conforman las subestaciones más representativas del Sistema Nacional de Transmisión (SNT), de igual manera se plantea la asignación de códigos para la fácil identificación y ubicación de los mismos, mediante una base de datos creada en Access, la misma que sirve para el almacenamiento de la información de los elementos del sistema. [4-6]

Con la base de datos creada se programa un script, utilizando el software computacional Matlab, que permita leer y disponer de la información ingresada en dicha base, además se ingresa el estado de los equipos de seccionamiento de la subestación, con el fin de determinar el estado operativo de cada uno de los elementos del sistema de potencia ingresado. [6-9]

El programa se realiza de tal manera que acepte el ingreso de cualquier subestación del SNT, lo que resulta sumamente conveniente ya que puede ser utilizado para una futura modelación de todo el SSEE. [9-11] 
El presente estudio tiene como objetivo desarrollar una guía de estilo mediante un programa que permita identificar al usuario del mismo la ubicación, especificaciones técnicas y estado operativo de cada uno de los elementos del sistema de potencia a ser analizado.

\section{Materiales y métodos.}

Con la finalidad de obtener un sistema homologado para dar nomenclatura a los equipos y elementos a considerar de las subestaciones seleccionadas del Sistema Nacional de Transmisión, se listan las normas generales a seguir en todos los casos:

- Todos los códigos deben escribirse en letras mayúsculas.

- $\quad$ No se incluirá la letra “ $\tilde{N}$ ”, en su lugar se utilizará la "N".

- $\quad$ No se admite el uso de tildes.

- Para la escritura se utilizará el tipo de letra “Consolas” tamaño 10.

- Para incluir espacios en blanco dentro del código se utilizará el guion bajo “_”.

- $\quad$ En caso de requerir menos caracteres de los disponibles, se llenará los caracteres restantes con el signo de punto “.”, los puntos se pondrá luego del nombre.

- $\quad$ Para separar un campo de otro, dentro del mismo código, se utilizará el guion medio “_“.

- Para la asignación de códigos se aceptan únicamente caracteres alfanuméricos, para el caso de espacios en blanco, espacios vacíos y separaciones, se utilizarán “_”, “"” Y “_.", respectivamente.

- $\quad$ En cuanto a la Jerarquía, como norma general se establece el siguiente orden, para cuando aplique el caso:

○ Ubicación geográfica: área y zona.

○ Nombre de la Central o Subestación. 
- Nivel de Voltaje.

○ Número de Bahía.

○ Nombre del Equipo.

○ Otros parámetros de identificación.

\section{Nomenclatura para áreas y zonas}

\section{Area}

Se entiende por área a la delimitación geográfica concesionada a una empresa de distribución eléctrica y que está comprendida por varias zonas.

El código del área estará conformado por 10 caracteres, 4 correspondientes a la palabra distribución, un carácter de separación correspondiente al guion medio “_“y los últimos 5 a la orientación norte, sur, este u oeste. Este criterio se establece a manera de ejemplo en la figura 1.

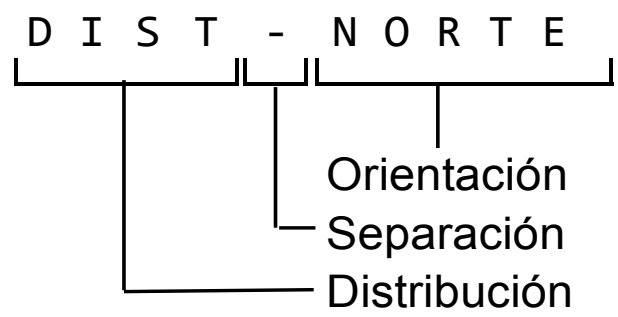

Figura 1 Codificación de Áreas

Por lo tanto se resume en la tabla 1 la codificación para las diferentes áreas. 
Guía de estilo para sistemas eléctricos de potencia

Tabla 1 Códigos para Áreas

\begin{tabular}{cll} 
No. & Nombre del Area & Código \\
\hline 1. & Distribución Norte & DIST-NORTE \\
2. & Distribución Sur & DIST-SUR \\
3. & Distribución Este & DIST-ESTE \\
4. & Distribución Oeste & DIST-OESTE
\end{tabular}

\section{Zona}

Es entiende por zona a la delimitación geográfica a la que sirve una subestación de distribución, la cual es alimentada por una red primaria. El conjunto de varias zonas conforma un área.

El código de la zona estará conformado por 10 caracteres, 4 correspondientes a la región del país, un caracter de separación correspondiente al guion medio “-_, y los 5 últimos a la orientación norte, sur, este u oeste. Este criterio se establece en la siguiente figura 2.

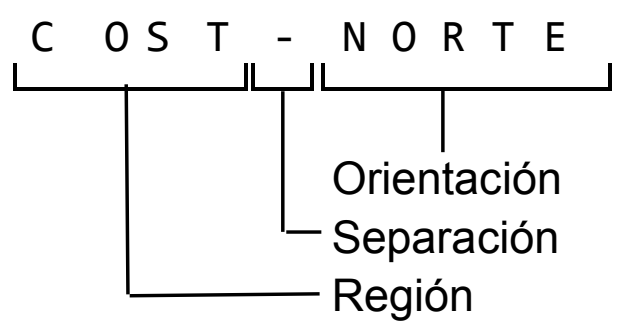

Figura 2. 2 Codificación de Zonas

En la tabla 2.2 se presenta la codificación para las diferentes zonas. 
Tabla 2. 2 Códigos para Zonas

\begin{tabular}{cll} 
No. & Nombre de la Zona & Código \\
\hline 1. & Costa Norte & COST-NORTE \\
2. & Costa Sur & COST-SUR.. \\
3. & Costa Este & COST-ESTE. \\
4. & Costa Oeste & COST-OESTE
\end{tabular}

\section{Nomenclatura para subestaciones del sistema}

Para la nomenclatura de las subestaciones del sistema se contará con 8 caracteres, que serán utilizados según las siguientes guías:

- Para los nombres que consten de artículos como: el, la, las, los; se utilizará la primera letra del artículo seguido por el sustantivo del nombre, sin dejar espacios o caracteres de separación. Como ejemplo, para Las Peñas se utilizará “LPENAS”.

- Si el código del nombre no completa los 8 caracteres, se rellenará los sobrantes con el signo de punto “." Siguiendo con el ejemplo anterior "LPENAS".

- Para títulos como: San, Santo, Santa, Don, Doña, etc; se utilizará la primera letra del título seguido por el sustantivo del nombre, sin dejar espacios o caracteres de separación. Como ejemplo, para San Nicolás se utilizará: "SNICOLAS".

- Nombres conformados por dos palabras se utilizarán las tres primeras letras de la primera palabra, un caracter de espacio “" y las cuatro primeras letras de la segunda palabra. Como por ejemplo, para Cuatro Colinas se utilizará: “CUA_COLI”.

- Nombres conformados con números ordinales como: I, II, III, etc. Estos se colocarán al final del código, disminuyendo, de ser necesario, el número de caracteres ocupados por la 
penúltima palabra. Como por ejemplo, para Los Laureles I será: “LLAURELI”; para los Los Laureles II será: “LLAUREII”.

De esta manera la codificación para las diferentes subestaciones se tendrá tal como se muestra en la tabla 2.3.

Tabla 2. 3 Códigos para Subestaciones

\begin{tabular}{clll} 
No. & Identificación de Campo & Identificación Externa & Código \\
\hline 1. & STATION A & SAN NICOLÁS & SNICOLAS \\
2. & STATION B & LAS PEÑAS & LPENAS.. \\
3. & STATION C & ÁLVARO ENRÍQUEZ & ALV_ENRI \\
4. & STATION D & CUATRO COLINAS & CUA_COLI \\
5. & STATION E & LOS LAURELES I & LLAURELI \\
6 & STATION F & LOS LAURELES II & LLAUREII
\end{tabular}

\section{Nomenclatura para voltajes del sistema}

Según los niveles de voltaje existentes en el sistema de subtransmisión y distribución, 138, $69,46,22.8,13.8$ y $6.3 \mathrm{kV}$, se utilizará la siguiente nomenclatura conformada por cuatro caracteres que los identifiquen, tal como se muestra en la tabla 2.4 .

Tabla 2. 4 Códigos para Niveles de Voltaje

\begin{tabular}{llrr} 
No. & Sistema & Nivel de Voltaje & \multicolumn{1}{c}{ Código } \\
\hline 1. & SUBTRANSMISIÓN & $138 \mathrm{kV}$ & $138 \mathrm{~K}$ \\
2. & SUBTRANSMISIÓN & $69 \mathrm{kV}$ & $69 \mathrm{KV}$ \\
3. & SUBTRANSMISIÓN & $46 \mathrm{kV}$ & $46 \mathrm{KV}$ \\
4. & DISTRIBUCIÓN & $22.8 \mathrm{kV}$ & $23 \mathrm{KV}$ \\
5. & DISTRIBUCIÓN & $13.8 \mathrm{kV}$ & $13 \mathrm{KV}$ \\
6. & DISTRIBUCIÓN & $6.3 \mathrm{kV}$ & $6 \mathrm{KV}$.
\end{tabular}




\section{Nomenclatura para las bahías}

Para la identificación de la bahía en una subestación, se cuenta con 7 caracteres, en los cuales se determina la identificación de la subestación, de la bahía y el número de las mismas, asignados según se muestra en la figura 2.3.

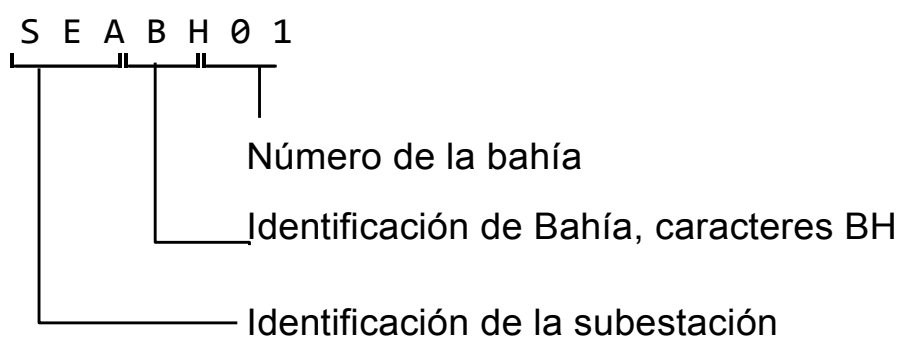

Figura 2. 3 Codificación para Bahías

\section{Nomenclatura para protocolos de comunicación}

Para los protocolos de comunicación se asignan tres caracteres. Según los protocolos existentes en el sistema, para lo cual se tiene la asignación mostrada en la tabla 2.5.

Tabla 2. 5 Códigos para Protocolos de Comunicación

\begin{tabular}{cll} 
No. & Protocolo & Código \\
\hline 1. & MODBUS & MOD \\
2. & IEC 61850 & IEC \\
3. & DNP 3.0 & DNP \\
4. & ICCP & ICC
\end{tabular}

\section{Nomenclatura para barras del sistema}

En el código para las barras del sistema se utilizarán 20 caracteres, siguiendo la jerarquía de la nomenclatura, para lo cual se identificará primeramente la subestación a la que pertenecen, luego el nivel de voltaje, la identificación de la barra en sí y de aplicar en la configuración de la 
subestación, se identifica si es una barra principal o de transferencia. La asignación de los campos se muestra en la figura 2.4 .

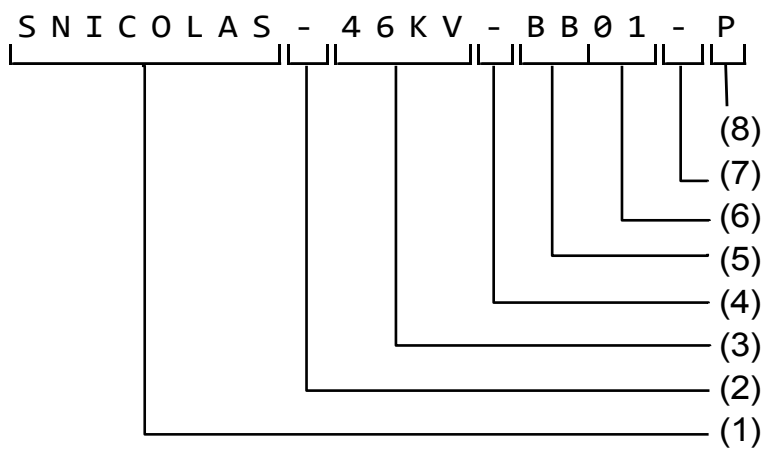

Figura 2. 4 Codificación para Barras del Sistema

Los números del esquema anterior, corresponden a los campos que se asignan según la tabla 2.6.

Tabla 2. 6 Asignación de Campos para Código en las Barras del Sistema

\begin{tabular}{|c|c|c|c|}
\hline Sampo & Descripción & $\begin{array}{c}\# \\
\text { de Caracteres }\end{array}$ & Caracteres Válidos \\
\hline (1) & $\begin{array}{l}\text { Nombre de la Subestación a } \\
\text { la que pertenece }\end{array}$ & 8 & Establecidos en la sección 3.3 \\
\hline (2) & Caracter de separación & 1 & “-“ \\
\hline (3) & Nivel de Voltaje & 4 & Establecidos en la sección 3.4 \\
\hline (4) & Caracter de separación & 1 & “-“ \\
\hline (5) & $\begin{array}{l}\text { Identificación del Elemento } \\
\text { Barra }\end{array}$ & 2 & $\mathrm{BB}=$ Barra \\
\hline (6) & Secuencial de la Barra & 2 & Números del 01 al 99 \\
\hline (7) & Caracter de separación & 1 & \\
\hline (8) & Tipo de Barra & 1 & $\begin{array}{l}\mathrm{P}=\text { Principal } \\
\mathrm{T}=\text { Transferencia }\end{array}$ \\
\hline
\end{tabular}

Para resumir la nomenclatura planteada en el presente inciso se toma como ejemplo los diagramas mostrados en las figuras 2.5 y 2.6. 


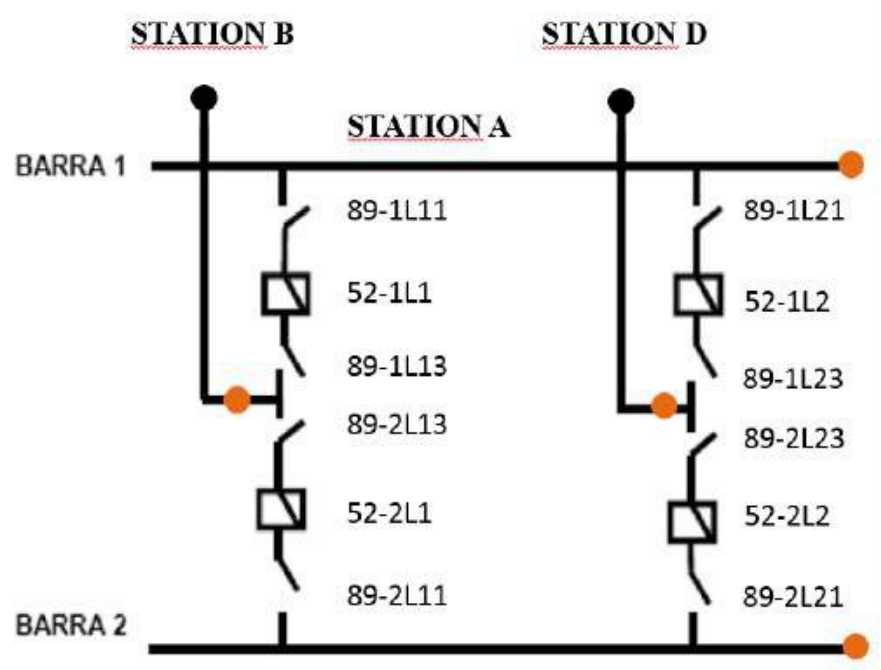

Figura 2. 5 Diagrama Unifilar Subestación A

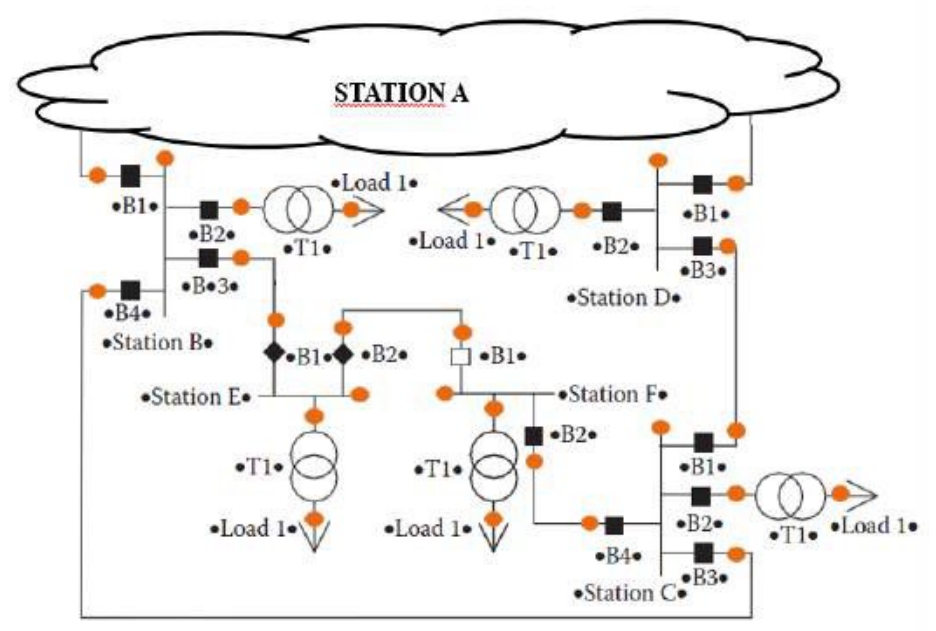

Figura 2. 6 Diagrama Unifilar Subestaciones B a la F

De lo que se puede acotar que para las barras de los diagramas de las figuras 2.5 y 2.6 les corresponde los códigos mostrados en la tabla 2.7. 
Guía de estilo para sistemas eléctricos de potencia

Tabla 2. 7 Códigos para las Barras de las Subestaciones A, B, C, D, E Y F

\begin{tabular}{cllccc} 
No. & $\begin{array}{l}\text { Identificación } \\
\text { de Campo }\end{array}$ & \multicolumn{1}{c}{ Subestación } & $\begin{array}{c}\text { Nivel de } \\
\text { Voltaje }\end{array}$ & Secuencial & Código \\
\hline 1. & STATION A & SAN NICOLAS & $46 \mathrm{kV}$ & 01 & SNICOLAS-46KV-BB01-P \\
2. & STATION A & SAN NICOLAS & $46 \mathrm{kV}$ & 02 & SNICOLAS-46KV-BB02-P \\
3. & STATION B & LAS PEÑAS & $46 \mathrm{kV}$ & 01 & LPENAS..-46KV-BB01-P \\
4. & STATION C & ÁLVARO ENRIQUEZ & $46 \mathrm{kV}$ & 01 & ALV_ENRI-46KV-BB01-P \\
5. & STATION D & CUATRO COLINAS & $46 \mathrm{kV}$ & 01 & CUA_COLI-46KV-BB01-P \\
6. & STATION E & LOS LAURELES I & $46 \mathrm{kV}$ & 01 & LLAURELI-46KV-BB01-P \\
7. & STATION F & LOS LAURELES II & $46 \mathrm{KV}$ & 01 & LLAUREII-46KV-BB01-P
\end{tabular}

\section{Nomenclatura para interruptores y seccionadores}

En el código para los elementos de maniobra (interruptores y seccionadores) se utilizarán 40 caracteres. Siguiendo la jerarquía establecida, los campos se asignan como se muestra en la figura 2.7 .

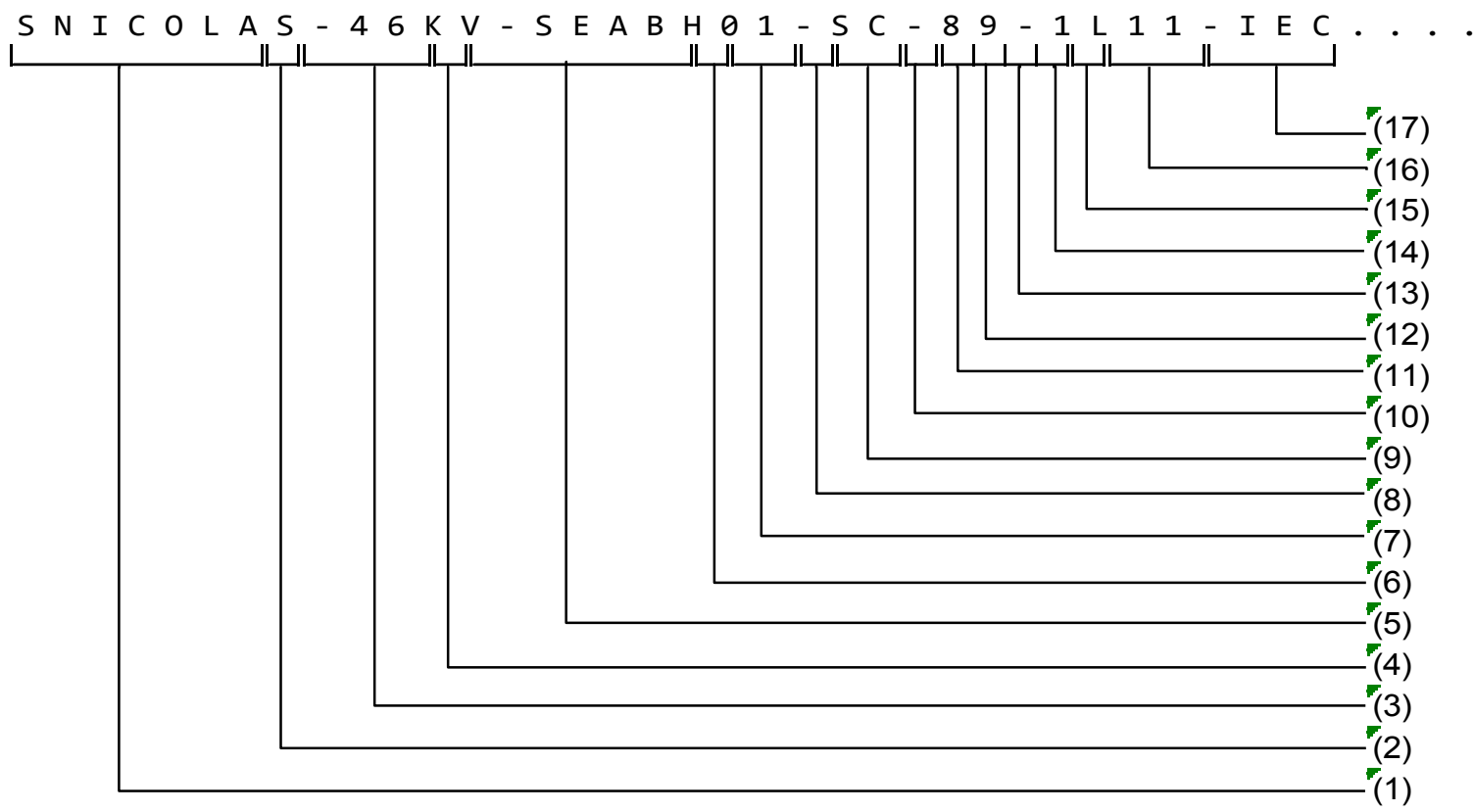

Figura 2. 7 Codificación para Interruptores y Seccionadores 
En el esquema anterior, los números corresponden a los campos listados en la tabla 2.8.

Tabla 2. 8 Asignación de campos en el código de elementos de maniobra

\begin{tabular}{|c|c|c|c|}
\hline $\begin{array}{l}\text { Campo } \\
\text { No. }\end{array}$ & Descripción & $\begin{array}{c}\text { \# de } \\
\text { Caracteres }\end{array}$ & Caracteres Válidos \\
\hline (1) & $\begin{array}{l}\text { Nombre de la Subestación a la } \\
\text { que pertenece }\end{array}$ & 8 & Establecidos en la sección 3.3 \\
\hline (2) & Caracter de separación & 1 & “-“ \\
\hline (3) & Nivel de Voltaje & 4 & Establecidos en la sección 3.4 \\
\hline (4) & Caracter de separación & 1 & “-“ \\
\hline (5) & Bahía en la que está ubicado & 7 & Establecidos en la sección 3.5 \\
\hline (6) & Caracter de separación & 1 & “-“ \\
\hline (7) & Tipo de Elemento & 2 & $\begin{array}{l}\text { SC }=\text { seccionador } \\
\mathrm{IT}=\text { interruptor }\end{array}$ \\
\hline (8) & Caracter de separación & 1 & “-“ \\
\hline (9) & Código ANSI del elemento & 2 & $\begin{array}{l}52=\text { interruptor } \\
89=\text { seccionador }\end{array}$ \\
\hline (10) & Caracter de separación & 1 & “-“ \\
\hline (11) & $\begin{array}{l}\text { Numeración del interruptor } \\
\text { principal }\end{array}$ & 1 & Números del 1 al 9 \\
\hline (12) & $\begin{array}{l}\text { Identificación del circuito para } \\
\text { línea o transformación }\end{array}$ & 1 & $\begin{array}{l}\mathrm{L}=\text { línea } \\
\mathrm{T}=\text { Transformador }\end{array}$ \\
\hline (13) & $\begin{array}{l}\text { Numeración de la línea o } \\
\text { transformador }\end{array}$ & 1 & Números del 1 al 9 \\
\hline (14) & $\begin{array}{l}\text { Numeración propia del elemento. } \\
\text { Si se trata del interruptor principal } \\
\text { se consigna "0" }\end{array}$ & 1 & Números del 0 al 9 \\
\hline (15) & Caracter de separación & 1 & “-“ \\
\hline (16) & $\begin{array}{l}\text { Protocolo utilizado por el } \\
\text { elemento }\end{array}$ & 3 & Establecidos en la sección 3.6 \\
\hline (17) & $\begin{array}{l}\text { Espacios libres para futuros } \\
\text { requerimientos }\end{array}$ & 4 & “...." \\
\hline
\end{tabular}

Continuando con el ejemplo de los diagramas planteados en las figuras 2.5 y 2.6, se les asigna los códigos correspondientes a los interruptores y seccionadores en la tabla 2.9.

Tabla 2. 9 Ejemplo de Codificación de Elementos de Maniobra 
Guía de estilo para sistemas eléctricos de potencia

\begin{tabular}{|c|c|c|c|}
\hline No. & Subestación & $\begin{array}{l}\text { Identificación } \\
\text { de Campo }\end{array}$ & Código \\
\hline 1 & SAN NICOLÁS & 89-1L11 & SNICOLAS-46KV-SEABH01-SC-89-1L11-IEC. \\
\hline 2 & SAN NICOLÁS & 52-1L1 & SNICOLAS-46KV-SEABH01-IT-52-1L10-IEC.... \\
\hline 3 & SAN NICOLÁS & 89-1L13 & SNICOLAS-46KV-SEABH01-SC-89-1L13-IEC.... \\
\hline 4 & SAN NICOLÁS & 89-2L11 & SNICOLAS-46KV-SEABH01-SC-89-2L11-IEC.... \\
\hline 5 & SAN NICOLÁS & 52-2L1 & SNICOLAS-46KV-SEABH01-IT-52-2L10-IEC.... \\
\hline 6 & SAN NICOLÁS & 89-2L13 & SNICOLAS-46KV-SEABH01-SC-89-2L13-IEC.... \\
\hline 7 & SAN NICOLÁS & 89-1L21 & SNICOLAS-46KV-SEABH01-SC-89-1L21-IEC.... \\
\hline 8 & SAN NICOLÁS & $52-1 \mathrm{~L} 2$ & SNICOLAS-46KV-SEABH01-IT-52-1L20-IEC.... \\
\hline 9 & SAN NICOLÁS & 89-1L23 & SNICOLAS-46KV-SEABH01-SC-89-1L23-IEC.... \\
\hline 10 & SAN NICOLÁS & $89-2 L 21$ & SNICOLAS-46KV-SEABH01-SC-89-2L21-IEC.... \\
\hline 11 & SAN NICOLÁS & $52-2 \mathrm{~L} 2$ & SNICOLAS-46KV-SEABH01-IT-52-2L20-IEC... \\
\hline 12 & SAN NICOLÁS & 89-2L23 & SNICOLAS-46KV-SEBBH01-SC-89-2L23-IEC... \\
\hline 13 & LAS PEÑAS & B1 & LPENAS..-46KV-SEBBH01-IT-52-1L10-IEC.... \\
\hline 14 & LAS PEÑAS & B2 & LPENAS..-46KV-SEBBH01-IT-52-1L20-IEC.... \\
\hline 15 & LAS PEÑAS & B3 & LPENAS..-46KV-SEBBH01-IT-52-1L30-IEC.... \\
\hline 16 & LAS PEÑAS & B4 & LPENAS..-46KV-SECBH01-IT-52-1L40-IEC.... \\
\hline 17 & ÁLVARO ENRÍQUEZ & B1 & ALV_ENRI-46KV-SECBH01-IT-52-1L10-IEC.... \\
\hline 18 & ÁLVARO ENRÍQUEZ & B2 & ALV_ENRI-46KV-SECBH01-IT-52-1L20-IEC.... \\
\hline 19 & ÁLVARO ENRÍQUEZ & B3 & ALV_ENRI-46KV-SECBH01-IT-52-1L30-IEC.... \\
\hline 20 & ÁLVARO ENRÍQUEZ & B4 & ALV_ENRI-46KV-SECBH01-IT-52-1L40-IEC.... \\
\hline 21 & CUATRO COLINAS & B1 & CUA_COLI-46KV-SEDBH01-IT-52-1L10-IEC.... \\
\hline 22 & CUATRO COLINAS & B2 & CUA COLI-46KV-SEDBH01-IT-52-1L20-IEC.... \\
\hline 23 & CUATRO COLINAS & B3 & CUA_COLI-46KV-SEDBH01-IT-52-1L30-IEC.... \\
\hline 24 & LOS LAURELES I & B1 & LLAURELI-46KV-SEDBH01-IT-52-1L10-IEC.... \\
\hline 25 & LOS LAURELES I & B2 & LLAURELI-46KV-SEDBH01-IT-52-1L20-IEC.... \\
\hline 26 & LOS LAURELES II & B1 & LLAUREII-46KV-SEDBH01-IT-52-1L10-IEC.... \\
\hline 27 & LOS LAURELES II & B2 & LLAUREII-46KV-SEDBH01-IT-52-1L20-IEC.... \\
\hline
\end{tabular}

\section{Nomenclatura para switchs de interruptores y seccionadores}

Para la codificación de los switchs de los elementos de maniobra se establece cuarenta caracteres. Para la asignación de los campos, se toma como base la asignación de interruptores, con las debidas modificaciones para identificar que se trata de switchs, tal como se muestra la asignación en la figura 2.8 . 


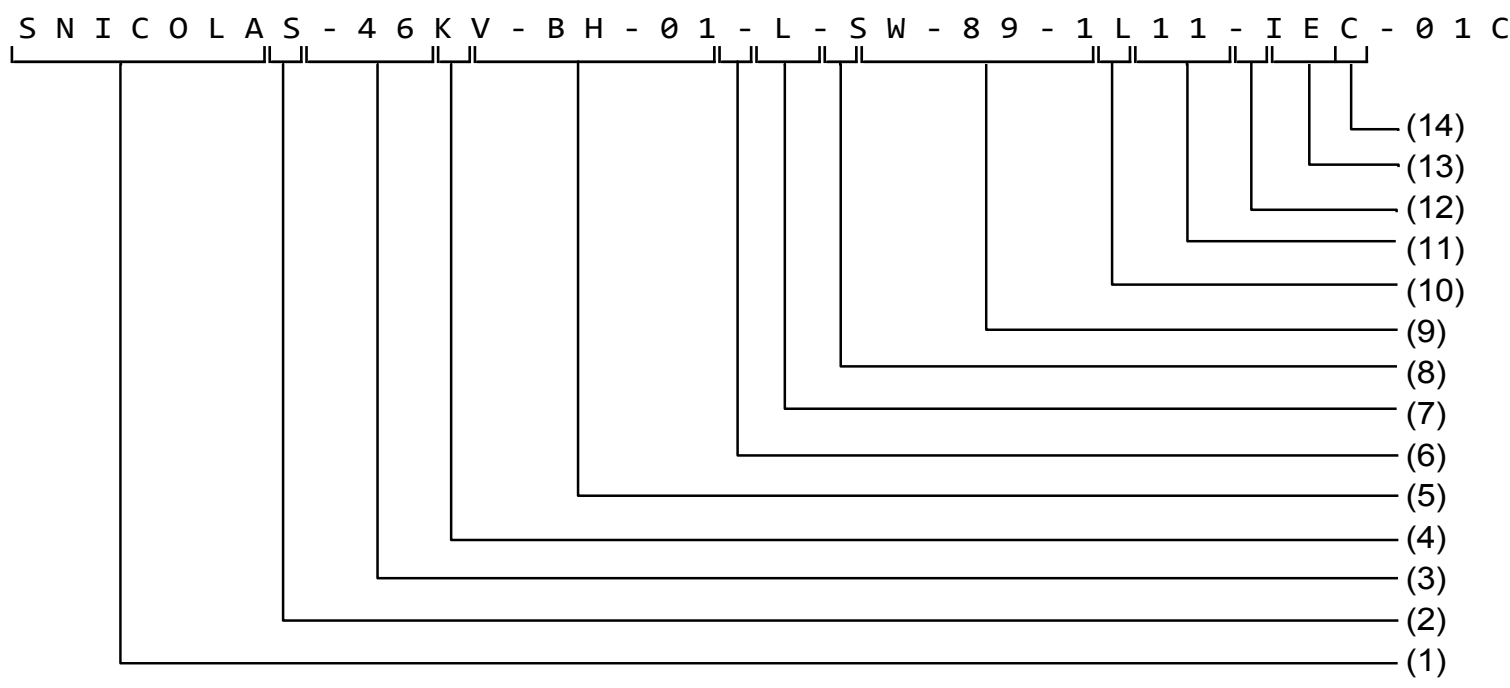

Figura 2. 8 Codificación para Switchs en Elementos de Maniobra

En el esquema anterior, los números corresponden a los campos listados en la tabla 2.10.

Tabla 2. 10 Asignación de campos en el código para switchs de elementos de maniobra

\begin{tabular}{|c|c|c|c|}
\hline $\begin{array}{l}\text { Campo } \\
\text { No. }\end{array}$ & Descripción & $\begin{array}{c}\text { \# de } \\
\text { Caracteres }\end{array}$ & $\begin{array}{l}\text { Caracteres } \\
\text { Válidos }\end{array}$ \\
\hline (1) & $\begin{array}{l}\text { Nombre de la Subestación a la } \\
\text { que pertenece }\end{array}$ & 8 & $\begin{array}{l}\text { Establecidos en la } \\
\text { sección } 3.3\end{array}$ \\
\hline$(2)$ & Caracter de separación & 1 & “_“ \\
\hline (3) & Nivel de Voltaje & 4 & $\begin{array}{l}\text { Establecidos en la } \\
\text { sección } 3.4\end{array}$ \\
\hline (4) & Caracter de separación & 1 & “-“ \\
\hline (5) & $\begin{array}{l}\text { Bahía en la que está ubicado el } \\
\text { elemento de maniobra }\end{array}$ & 7 & $\begin{array}{l}\text { Establecidos en la } \\
\text { sección } 3.5\end{array}$ \\
\hline (6) & Caracter de separación & 1 & “-“ \\
\hline (7) & Tipo de Elemento & 2 & SW = switch \\
\hline (8) & Caracter de separación & 1 & “-“ \\
\hline (9) & $\begin{array}{l}\text { Código del elemento de } \\
\text { maniobra }\end{array}$ & 7 & $\begin{array}{l}\text { Establecidos en la } \\
\text { sección } 3.9\end{array}$ \\
\hline (10) & Caracter de separación & 1 & “-“ \\
\hline (11) & $\begin{array}{l}\text { Protocolo utilizado por el } \\
\text { elemento }\end{array}$ & 3 & $\begin{array}{l}\text { Establecidos en la } \\
\text { sección } 3.6\end{array}$ \\
\hline (12) & Caracter de separación & 1 & “-“ \\
\hline
\end{tabular}


Guía de estilo para sistemas eléctricos de potencia

\begin{tabular}{clcl}
$\begin{array}{c}\text { Campo } \\
\text { No. }\end{array}$ & \multicolumn{1}{c}{ Descripción } & \multicolumn{1}{c}{$\begin{array}{l}\text { \# de } \\
\text { Caracteres }\end{array}$} & \multicolumn{1}{c}{$\begin{array}{c}\text { Caracteres } \\
\text { Válidos }\end{array}$} \\
\cline { 1 - 1 } & $\begin{array}{l}\text { Numeración secuencial del } \\
\text { switch }\end{array}$ & 2 & $\begin{array}{l}\text { Números del 01 al } \\
99\end{array}$ \\
& & & $\begin{array}{l}\text { Normalmente } \\
\text { cerrado }=\mathrm{C}\end{array}$ \\
(14) & Estado normal del switch & 1 & $\begin{array}{l}\text { Normalmente } \\
\text { abierto }=0\end{array}$
\end{tabular}

\section{Nomenclatura para líneas de transmisión}

Para la codificación de las líneas de transmisión se utiliza 40 caracteres. Para la asignación de los campos, se sigue la jerarquía de codificación, con las debidas modificaciones para los parámetros relevantes en líneas de transmisión, tal como se muestra en la figura 2.9.

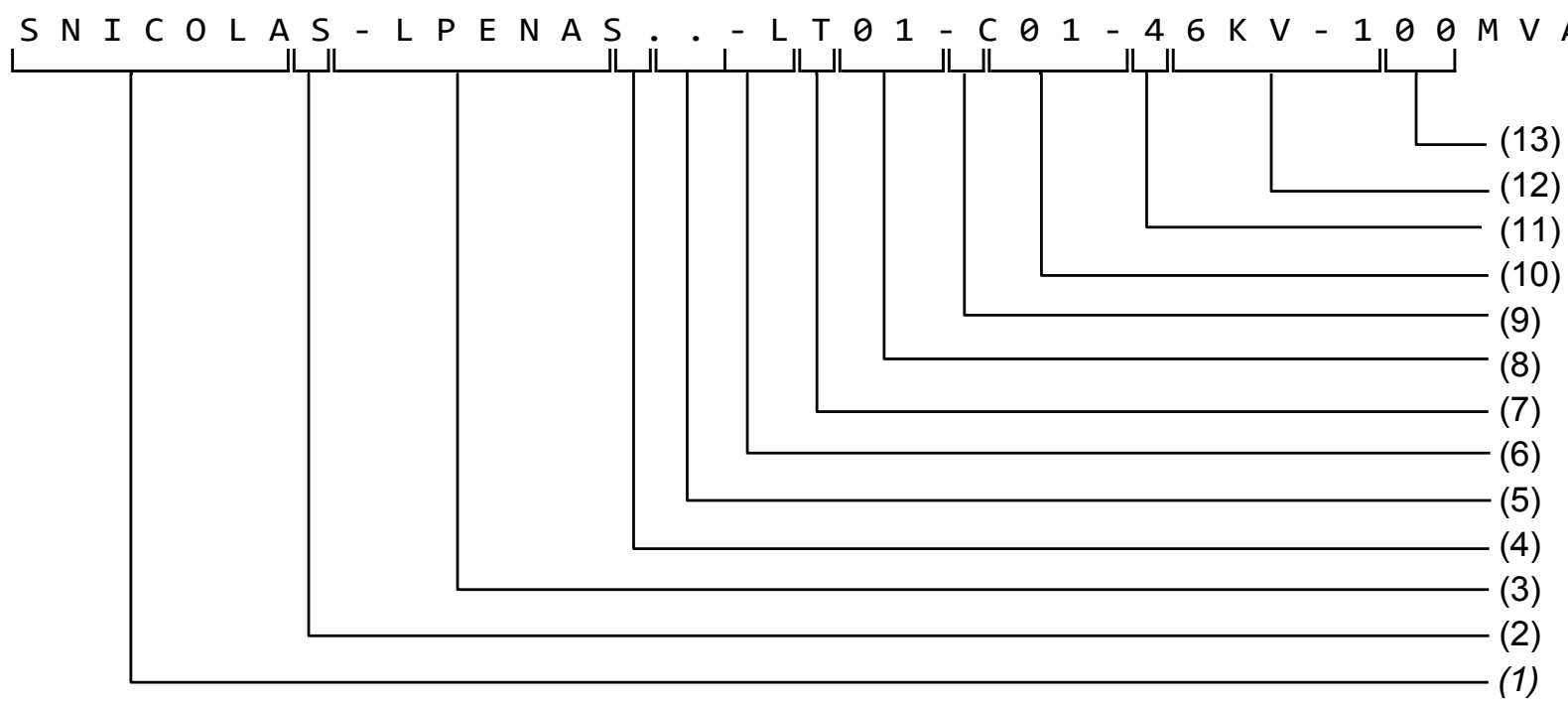

2. 9 Codificación para Líneas de Transmisión

En el esquema anterior, los números corresponden a los campos listados a en la tabla 2.11. 
Raul C. Ulloa De Souza; Alejandro J. Martinez-Peralta; Maria E. Moreno-Sanchez; Byron F. Chere-Quiñonez

Tabla 2. 11 Asignación de campos en el código para líneas de transmisión

\begin{tabular}{|c|c|c|c|}
\hline $\begin{array}{l}\text { Campo } \\
\text { No. }\end{array}$ & Descripción & $\begin{array}{c}\text { \# de } \\
\text { Caracteres }\end{array}$ & Caracteres Válidos \\
\hline (1) & $\begin{array}{l}\text { Nombre de la Subestación de } \\
\text { salida }\end{array}$ & 8 & Establecidos en la sección 3.3 \\
\hline (2) & Caracter de separación & 1 & “-“ \\
\hline (3) & $\begin{array}{l}\text { Nombre de la Subestación de } \\
\text { Ilegada }\end{array}$ & 8 & Establecidos en la sección 3.3 \\
\hline (4) & Caracter de separación & 1 & “-“ \\
\hline (5) & Tipo de Elemento & 2 & LT = Línea de Transmisión \\
\hline (6) & Secuencial del Elemento & 2 & $\begin{array}{l}\text { Número secuencial de la línea, } \\
\text { del } 01 \text { al } 99\end{array}$ \\
\hline (7) & Caracter de separación & 1 & "-“ \\
\hline (8) & Número de Circuito & 3 & $\begin{array}{l}\text { C seguido por dos números } \\
\text { del } 01 \text { al } 99\end{array}$ \\
\hline (9) & Caracter de separación & 1 & “-“ \\
\hline (10) & Nivel de Voltaje de la línea & 4 & Establecidos en la sección 3.4 \\
\hline (11) & Caracter de separación & 1 & $\begin{array}{l}\text { "-“ } \\
3 \text { números }\end{array}$ \\
\hline (12) & Capacidad de la línea & 5 & $\begin{array}{l}3 \text { caracteres para las unidades } \\
\text { en múltiplos de VA }\end{array}$ \\
\hline (13) & Espacios disponibles & 2 & “.." \\
\hline
\end{tabular}

Continuando con el ejemplo de los diagramas planteados en las figuras 2.5 y 2.6, se les asigna los códigos correspondientes a las líneas de transmisión en la tabla 2.12. 
Guía de estilo para sistemas eléctricos de potencia

Tabla 2. 12 Ejemplo de codificación de Líneas de transmisión

\begin{tabular}{|c|c|c|c|c|}
\hline No. & $\begin{array}{l}\text { Subestación } \\
\text { de Salida }\end{array}$ & $\begin{array}{l}\text { Subestación } \\
\text { de Llegada }\end{array}$ & Voltaie & Códiao \\
\hline 1 & SAN NICOLAS & $\begin{array}{l}\text { LAS PEÑAS } \\
\text { CUATRO }\end{array}$ & $46 \mathrm{kV}$ & SNICOLAS-LPENAS..-LT01-C01-46KV-100MVA.. \\
\hline 2 & SAN NICOLAS & $\begin{array}{l}\text { COLINAS } \\
\text { LOS }\end{array}$ & $46 \mathrm{kV}$ & SNICOLAS-CUA_COLI-LT01-C01-46KV-100MVA. \\
\hline 3 & LAS PEÑAS & $\begin{array}{l}\text { LAURELES I } \\
\text { LOS }\end{array}$ & $46 \mathrm{kV}$ & LPENAS..-LLAURELI-LT01-C01-46KV-100MVA.. \\
\hline 4 & $\begin{array}{l}\text { LOS LAURELES I } \\
\text { CUATRO }\end{array}$ & $\begin{array}{l}\text { LAURELES II } \\
\text { ALVARO }\end{array}$ & $46 \mathrm{kV}$ & LLAURELI-LLAUREII-LT01-C01-46KV-100MVA.. \\
\hline 5 & $\begin{array}{l}\text { COLINAS } \\
\text { ALVARO }\end{array}$ & $\begin{array}{l}\text { ENRIQUEZ } \\
\text { LOS }\end{array}$ & $46 \mathrm{kV}$ & CUA_COLI-ALV_ENRI-LT01-C01-46KV-100MVA.. \\
\hline 6 & ENRIQUEZ & LAURELES II & $46 \mathrm{kV}$ & ALV_ENRI-LLAUREII-LT01-C01-46KV-100MVA.. \\
\hline
\end{tabular}

\section{Nomenclatura para transformadores de potencia}

Para la codificación de los transformadores de potencia ubicados en las subestaciones se utiliza 40 caracteres. Para la asignación de los campos, se sigue la jerarquía de codificación, con las debidas modificaciones para identificar que se trata de transformadores, tal como se indica en la figura 2.10 .

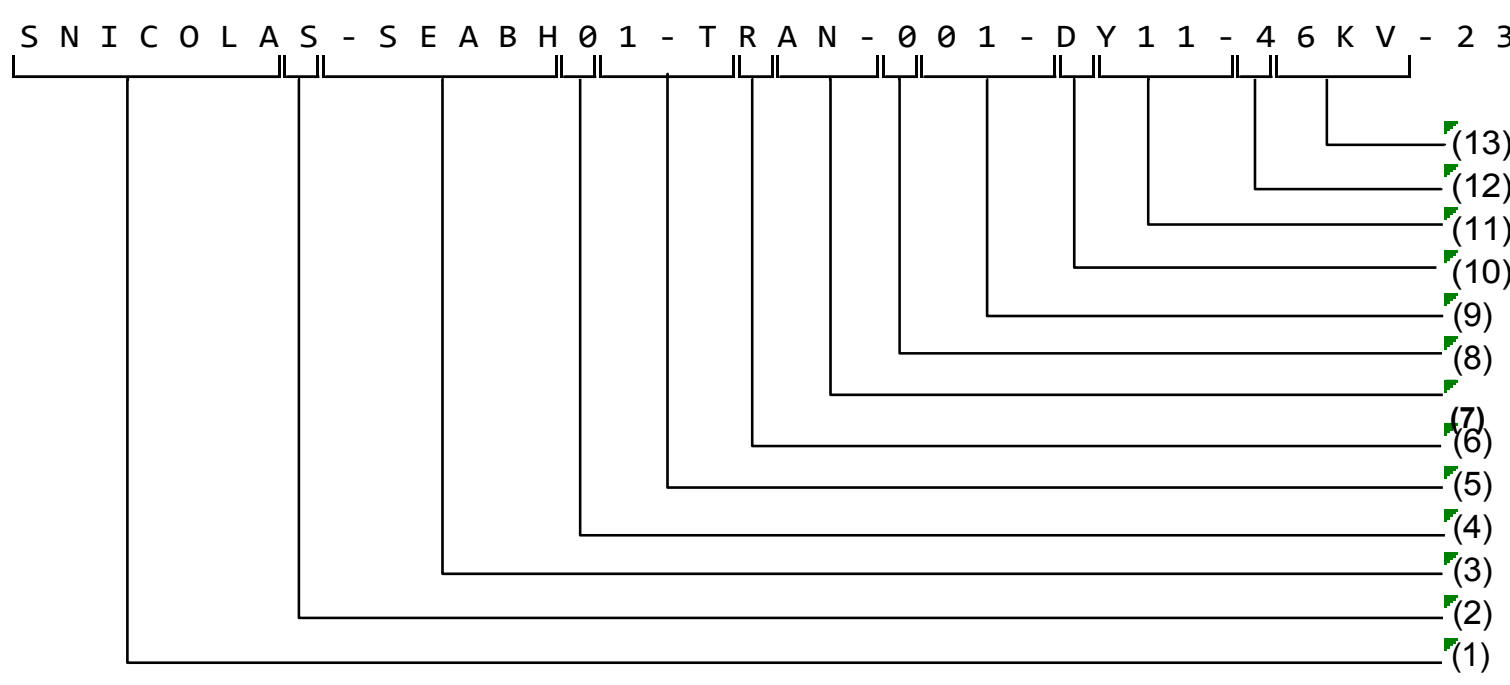

Figura 2.

10 Codificación para Transformadores de Potencia

En la tabla 2.13 se explica de manera detallada el orden jerárquico para la asignación de los campos del esquema mostrado en la figura anterior. 
Tabla 2. 13 Asignación de campos en el código para transformadores

\begin{tabular}{|c|c|c|c|}
\hline $\begin{array}{l}\text { Campo } \\
\text { No. }\end{array}$ & nacerinnión & $\begin{array}{c}\text { \# de } \\
\text { Caracteres }\end{array}$ & Carantarace Válidne \\
\hline (1) & $\begin{array}{l}\text { INomore de la suovestacion a } \\
\text { la que pertenece }\end{array}$ & 8 & Establecidos en la sección 3.3 \\
\hline (2) & Caracter de separación & 1 & “-“ \\
\hline (3) & Bahía en la que está ubicado & 7 & $\begin{array}{l}\text { Establecidos en la sección } 3.5 \\
\text { Si no aplica, consignar “...." }\end{array}$ \\
\hline (4) & Caracter de separación & 1 & “-“ \\
\hline (5) & Tipo de Elemento & 4 & TRAN = TRANSFORMADOR \\
\hline (6) & Caracter de separación & 1 & “-“ \\
\hline (7) & Secuencial del Transformador & 3 & Números del 001 al 999 \\
\hline (8) & Caracter de separación & 1 & “-“ \\
\hline & & & $\begin{array}{l}\text { Primer caracter: Conexión del } \\
\text { Primario }\end{array}$ \\
\hline (9) & Tipo de Conexión & 4 & $\begin{array}{l}\text { Segundo caracter: Conexión del } \\
\text { Secundario }\end{array}$ \\
\hline & & & $\begin{array}{l}\text { Tercero y Cuarto carácter: número } \\
\text { de la conexión }\end{array}$ \\
\hline (10) & Caracter de separación & 1 & “_“ \\
\hline (11) & Voltaje del primario & 4 & Establecidos en la sección 3.4 \\
\hline (12) & Caracter de separación & 1 & “_“ \\
\hline (13) & Voltaje del Secundario & 4 & Establecidos en la sección 3.4 \\
\hline
\end{tabular}

En la tabla 2.14 se asigna los códigos correspondientes a los transformadores de los diagramas planteados en las figuras 2.5 y 2.6, los cuales recibirían la siguiente codificación. 
Guía de estilo para sistemas eléctricos de potencia

Tabla 2. 14 Ejemplo de codificación de transformadores

\begin{tabular}{rl|ll} 
No. & \multicolumn{1}{c|}{ Subestación } & $\begin{array}{c}\text { Voltajes del } \\
\text { Transformador }\end{array}$ & \multicolumn{1}{c}{ Código } \\
\hline 1 & LAS PEÑAS & $46 / 23 \mathrm{kV}$ & LPENAS..-BH-01-T-TRAN-001-DY11-46KV-23KV \\
2 & ÁLVARO & $46 / 23 \mathrm{kV}$ & ALV_ENRI-BH-01-T-TRAN-001-DY11-46KV-23KV \\
& $\begin{array}{l}\text { ENRIQUEZ } \\
\text { CUATRO }\end{array}$ & & \\
& COLINAS & $46 / 23 \mathrm{kV}$ & CUA_COLI-BH-01-T-TRAN-001-DY11-46KV-23KV \\
4 & LOS LAURELES I & $46 / 13.8 \mathrm{kV}$ & LLAURELI-BH-01-T-TRAN-001-DY11-46KV-13KV \\
5 & LOS LAURELES II & $46 / 13.8 \mathrm{kV}$ & LLAUREII-BH-01-T-TRAN-001-DY11-46KV-113KV
\end{tabular}

\section{Nomenclatura para las cargas del sistema}

Para las cargas del sistema se utiliza una totalidad de 25 caracteres, siguiendo la jerarquía de la nomenclatura, los campos se asignan según el detalle dado en la figura 2.11.

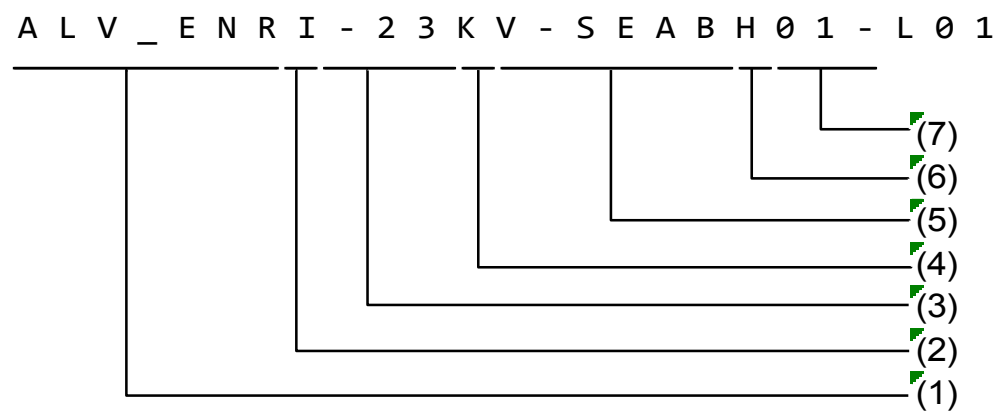

Figura 2. 11 Codificación para Cargas

En la tabla 2.15 se detalla a manera resumida la asignación de los números que corresponden a los campos para dar nomenclatura a las cargas del sistema.

Tabla 2. 15 Asignación de campos en el código para Cargas

\begin{tabular}{|c|c|c|c|}
\hline $\begin{array}{l}\text { Campo } \\
\text { No. }\end{array}$ & Descripción & $\begin{array}{c}\text { \# de } \\
\text { Caracteres }\end{array}$ & Caracteres Válidos \\
\hline$(1)$ & $\begin{array}{l}\text { Nombre de la Subestación a la que } \\
\text { pertenece }\end{array}$ & 8 & Establecidos en la sección 3.3 \\
\hline (2) & Caracter de separación & 1 & “-“ \\
\hline (3) & Nivel de Voltaje & 4 & Establecidos en la sección 3.4 \\
\hline (4) & Caracter de separación & 1 & “-“ \\
\hline (5) & Bahía en la que está ubicado & 7 & Establecidos en la sección 3.5 \\
\hline (6) & Caracter de separación & 1 & “-“ \\
\hline
\end{tabular}


Raul C. Ulloa De Souza; Alejandro J. Martinez-Peralta; Maria E. Moreno-Sanchez; Byron F. Chere-Quiñonez
Campo
No.
Descripción
\# de
Caracteres
Caracteres Válidos
(7) Tipo de Elemento y secuencia 3
$\mathrm{L}=$ carga
Números secuenciales del 01 al 99

En la tabla 2.16 se asigna los códigos correspondientes a las respectivas cargas de los diagramas planteados en las figuras 2.5 y 2.6 .

Tabla 2. 16 Ejemplo de Codificación para cargas del Sistema

\begin{tabular}{clcl} 
No. & \multicolumn{1}{c}{ Subestación } & $\begin{array}{c}\text { Voltaje de } \\
\text { la Carga }\end{array}$ & \multicolumn{1}{c}{ Código } \\
\hline 1 & LAS PEÑAS & $23 \mathrm{kV}$ & LPENAS..-23KV-SEABH01-L01 \\
2 & ÁLVARO ENRÍQUEZ & $23 \mathrm{kV}$ & ALV_ENRI-23KV-SEABH01-L01 \\
3 & CUATRO COLINAS & $23 \mathrm{kV}$ & CUA_COLI-23KV-SEABH01-L01 \\
4 & LOS LAURELES I & $13.8 \mathrm{kV}$ & LLAURELI-13KV-SEABH01-L01 \\
5 & LOS LAURELES II & $13.8 \mathrm{kV}$ & LLAUREII-13KV-SEABH01-L01
\end{tabular}

\section{Nomenclatura para unidades terminales remotas (RTU)}

Para la codificación de Unidades Terminales Remotas se asigna 16 caracteres para identificar en donde se encuentra la RTU y el protocolo que utiliza. En la figura 2.12 se detallan dichos códigos.

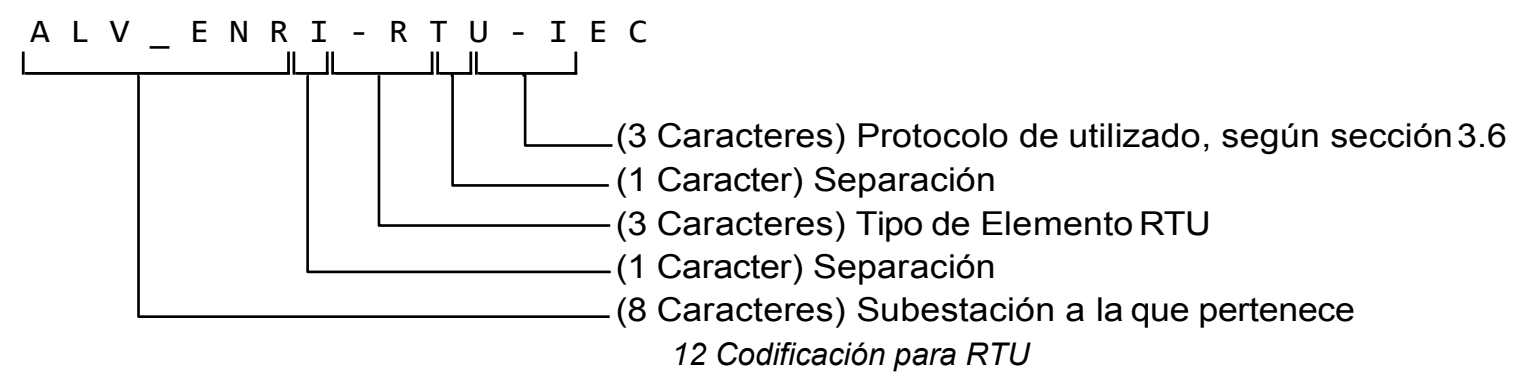

Figura 2.

\section{Nomenclatura para alarmas del sistema}

Para la nomenclatura de las alarmas se establece 40 caracteres, según la asignación planteada en la figura 2.13 . 
Guía de estilo para sistemas eléctricos de potencia

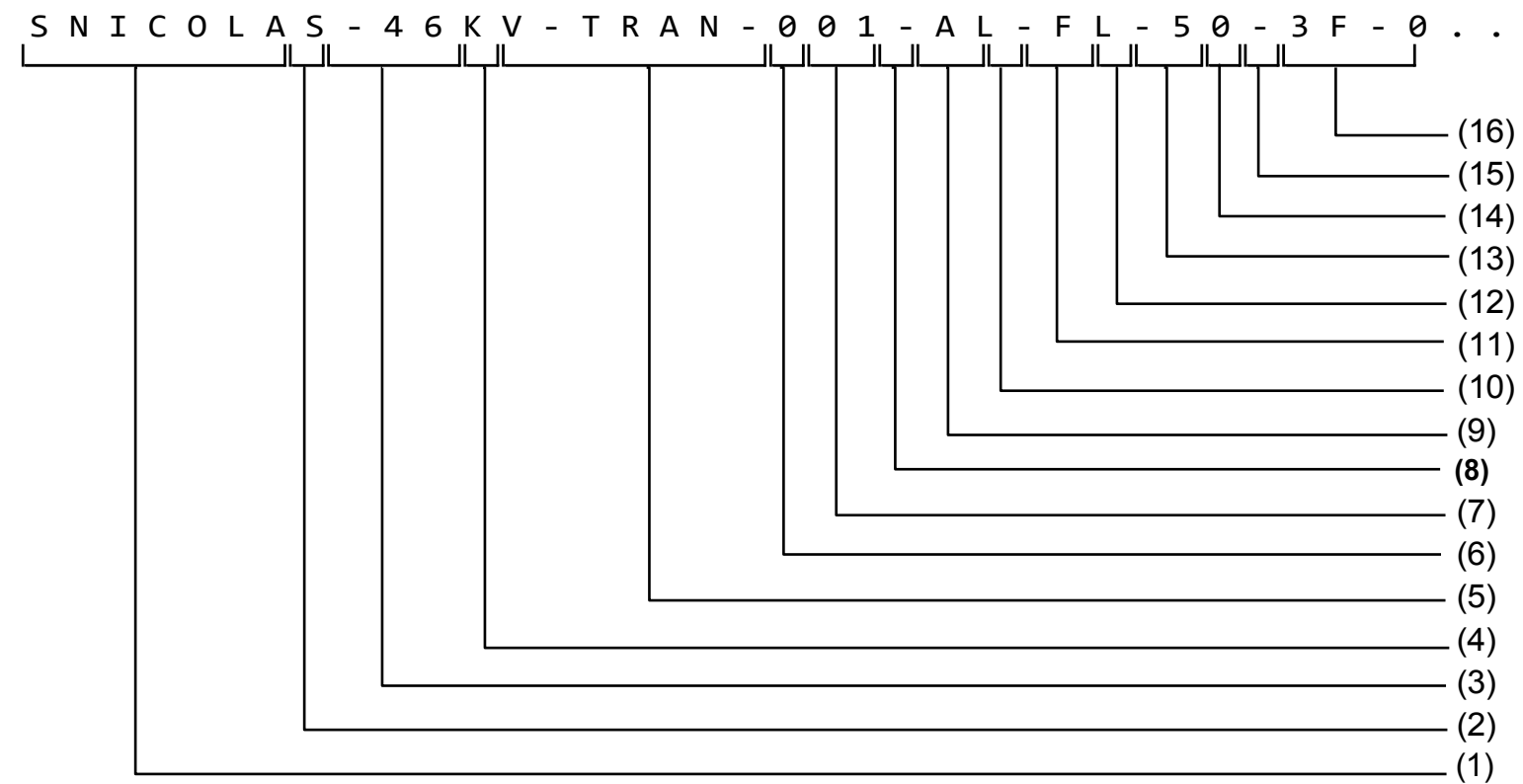

13 Codificación para Alarmas del Sistema

Figura 2.

En la tabla 2.17 se detalla a manera resumida la asignación de los números que corresponden a los campos para dar nomenclatura a las alarmas del sistema.

Tabla 2. 17 Asignación de campos en el código de alarmas

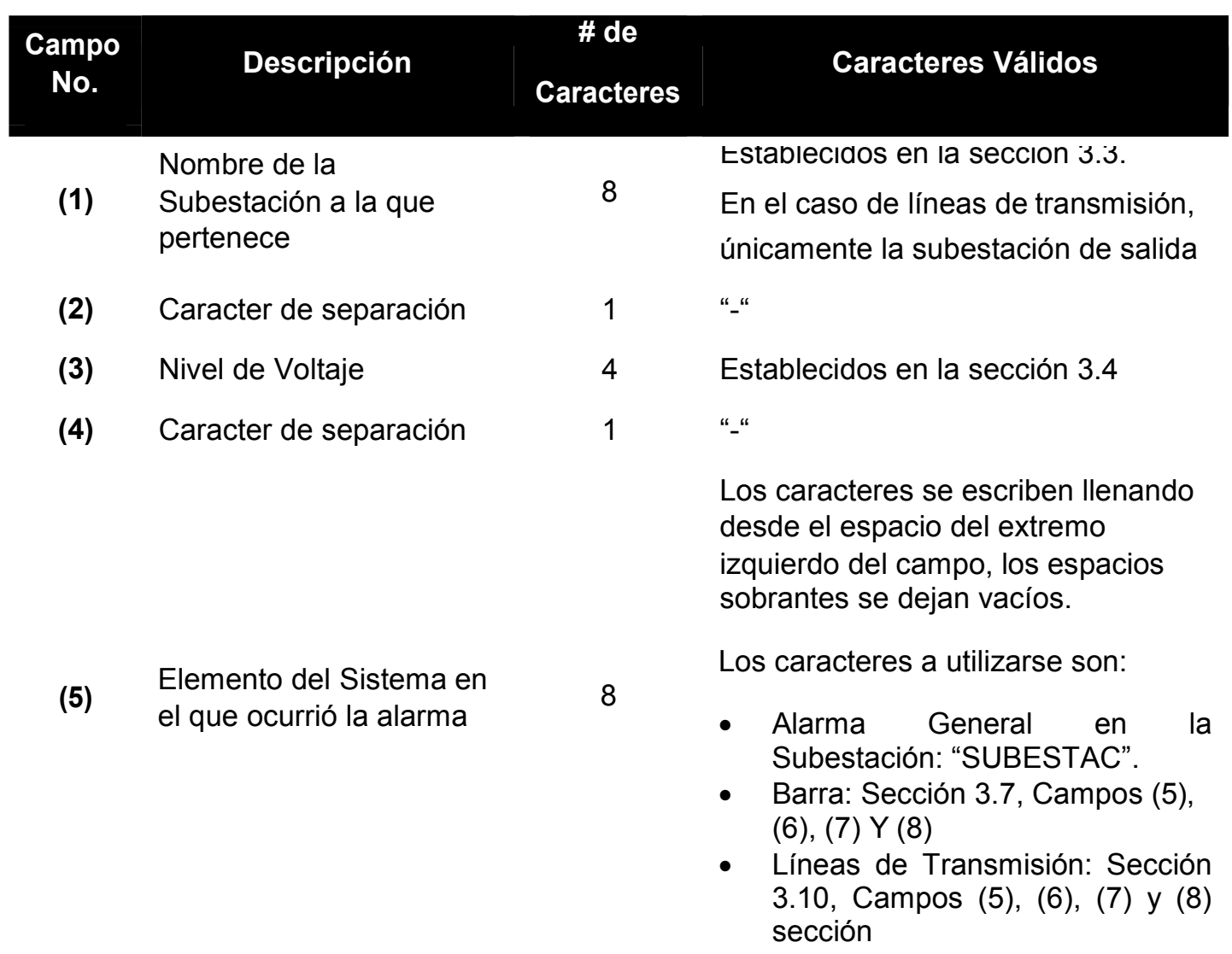

Pol. Con. (Edición núm. 9) Vol. 2, No 8, agosto 2017, pp. 362-405, ISSN: 2550 - 682X 


\begin{tabular}{|c|c|c|c|}
\hline $\begin{array}{c}\text { Campo } \\
\text { No. }\end{array}$ & Descripción & $\begin{array}{c}\text { \# de } \\
\text { Caracteres }\end{array}$ & Caracteres Válidos \\
\hline & & & $\begin{array}{l}\text { - } \quad \text { Transformadores: Sección 3.11, } \\
\text { Campos (5), (6), (7) } \\
\text { - Cargas: Sección 3.12, Campo } \\
\text { (7) }\end{array}$ \\
\hline (6) & Caracter de separación & 1 & “-“ \\
\hline (7) & Identificación de Alarma & 2 & $\mathrm{AL}=$ Alarma \\
\hline (8) & Caracter de separación & 1 & “-“ \\
\hline (9) & Tipo de Alarma & 2 & $\begin{array}{l}\mathrm{FL}=\text { por falla } \\
\mathrm{SO}=\text { por salida de operación }\end{array}$ \\
\hline (10) & Caracter de separación & 1 & “-“ \\
\hline
\end{tabular}

Los campos a continuación se llenan únicamente si la alarma ocurrió por falla en los elementos: barra, transformador, línea de transmisión y carga. Caso contrario se dejan vacíos

Código ANSI del relé de protección que se disparó en la falla

(12)

(13)

(14)

Caracter de separación
Número de fases en las
que ocurrió la falla
Caracter de separación
Fase o Fases en las que
ocurrió la falla

Espacios libres para futuros requerimientos
Dos números correspondientes al código ANSI del relé. Ejemplo:

2

50 = sobre corriente instantáneo

$51=$ sobre corriente temporizado

$59=$ sobre voltaje

1

$23 \mathrm{~F}=$ trifásica

$1 \mathrm{~F}=$ monofásica

1 "-"

$0=$ para trifásica

$1 \quad 1,2,3=$ para monofásica según corresponda a la fase medida

4 “..."

Con la finalidad de consolidar la codificación mostrada para alarmas del sistema, se presenta dos ejemplos prácticos:

- Una alarma por salida de operación en la subestación San Nicolás. 
- SNICOLAS-46KV-SUBESTAC-AL-SO- - - ...

- Una alarma por falla de sobrecorriente en la barra de la Subestación Cuatro Colinas.

○ CUA_COLI-46KV-BB01-P-AL-FL-50-3F-0...

\section{Nomenclatura para mediciones del sistema}

Para la nomenclatura de las mediciones se establece 40 caracteres, según la asignación detallada en la figura 2.14 .

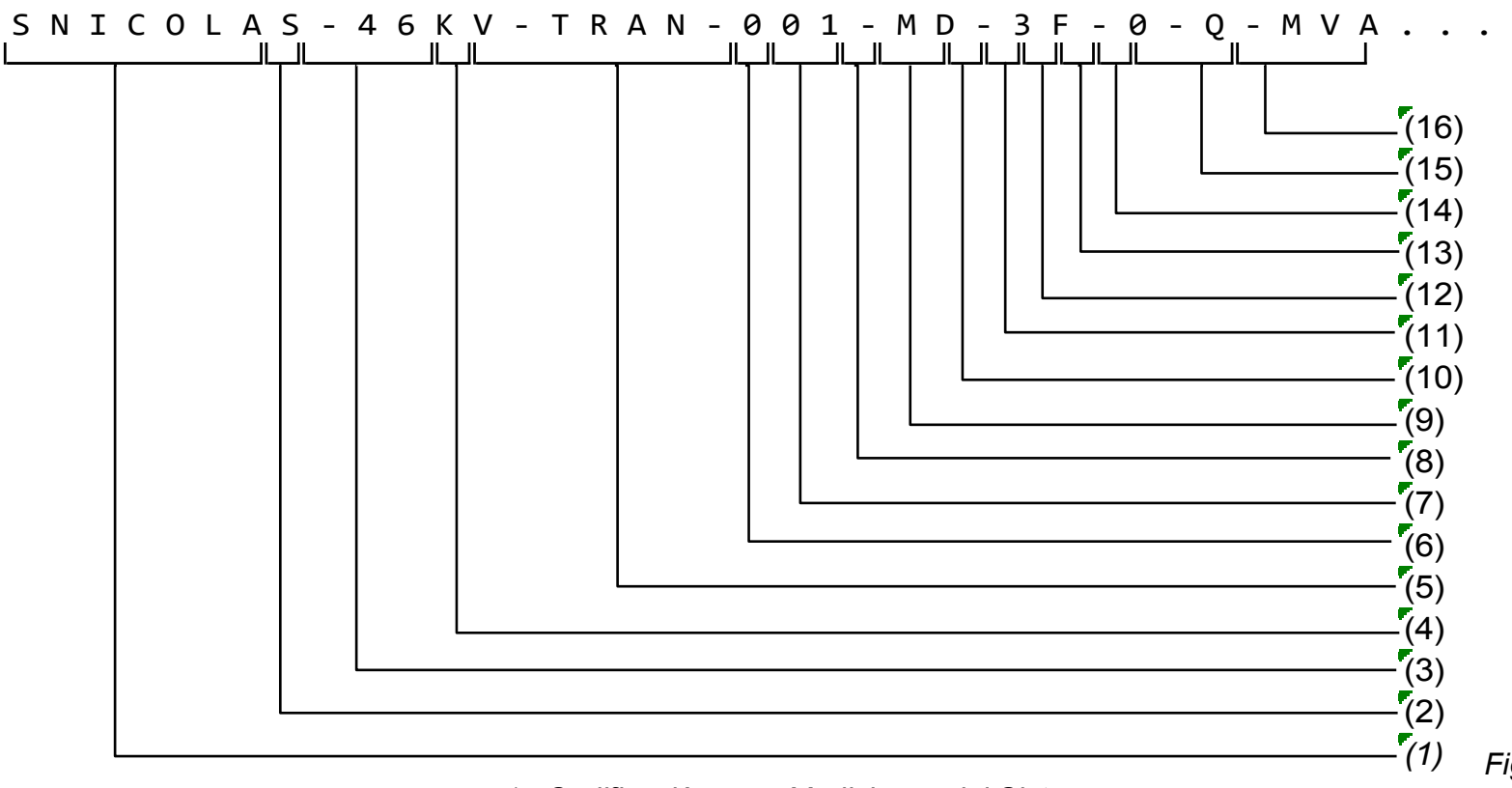

En el esquema anterior, los números corresponden a los campos listados en la tabla 2.18.

Tabla 2. 18 Asignación de campos en el código de mediciones

\begin{tabular}{llcl}
$\begin{array}{c}\text { Campo } \\
\text { No. }\end{array}$ & \multicolumn{1}{c}{ Descripción } & $\begin{array}{c}\text { \# de } \\
\text { Caracteres }\end{array}$ & \multicolumn{1}{c}{ Caracteres Válidos } \\
(1) & $\begin{array}{l}\text { Nombre de la Subestación a } \\
\text { la que pertenece }\end{array}$ & 8 & $\begin{array}{l}\text { Establecidos en la sección } 3.3 \\
\text { En el caso de líneas de transmisión, } \\
\text { únicamente la subestación de salida }\end{array}$ \\
(2) & Caracter de separación & 1 & "-“ \\
(3) & Nivel de Voltaje & 4 & Establecidos en la sección 3.4 \\
(4) & Caracter de separación & 1 & "-“
\end{tabular}




\section{Caracteres Válidos}

Los caracteres se escriben llenando desde el espacio del extremo izquierdo de campo, los espacios sobrantes se dejan vacíos.

(5) Elemento del Sistema al que pertenece la medición

(6) Caracter de separación

(7) Identificación de Medición

(8) Caracter de separación

(9) Número de Fases de la Medición

(10) Caracter de separación

(11) rase a la que currespurrue la medición

(12) Caracter de separación

(13) Tipo de medición

(14) Caracter de separación

(15) Unidades de Medición

(16) Espacios libres para futuros

1

2

1

2

1

1
- Los caracteres a utilizarse son:

- Barra: Sección 3.7, Campos (5), (6), (7) y (8)

- Líneas de Transmisión: Sección 3.10, Campos (5), (6), (7) y (8) sección

- Transformadores: Sección 3.11, Campos (5), (6), (7)

• “ Cargas: Sección 3.12, Campo (7)

$\mathrm{MD}=$ Medición

$3 F=$ trifásica

$1 \mathrm{~F}=$ Monofásica

“-“

$0=$ para trifásica

1, 2, 3 = para monofásica según corresponda a la fase medida

1 “-“

$\mathrm{P}=$ Potencia activa

$Q=$ Potencia Reactiva

$1 \quad V=$ Voltaje

$\mathrm{F}=$ Frecuencia

$\mathrm{I}=$ Corriente

1 “-“

Primer carácter, prefijo de múltiplo según el sistema internacional: $M=$ Mega; K=kilo

3 Segundo y Tercer carácter, unidad de medición: $V=$ Voltios, $A=$ Amperios, $\mathrm{HZ}=$ Hertz, $\mathrm{W}=$ Vatios, $V A=$ Voltio Amperios

$4 \quad$ "..."


Con la finalidad de consolidar la codificación mostrada para mediciones del sistema, se presenta dos ejemplos prácticos:

- Una medición de potencia trifásica, en MW, en la línea de salida 01 de la Subestación Las Peñas.

- LPENAS..-46KV-LT01-C01-MD-3F-0-P-MW....

- Una medición de corriente en la fase 1, en kA de la carga en la Subestación Álvaro Enríquez.

- ALV_ENRI-46KV-L01-MD-1F-1-I-KA.....

\section{Resultados.}

\section{Base de datos}

La base de datos del sistema se la realizó utilizando el software Microsoft Access atendiendo la guía establecida y detallada en la parte de Metodología y Métodos, dicha base de datos consta de las siguientes tablas:

- SOSUBS: Subestaciones del Sistema

- SOBARR: Barras del Sistema

- SOSNET: Niveles de Voltaje

- SOINTE: Interruptores y Seccionadores del Sistema

- SOSWCH: Interruptores y Seccionadores utilizados en las Funciones de Aplicación del SED

- SOALAR: Alarmas del Sistema

- SOMEDI: Mediciones del Sistema

- SOBAYS: Nombre de las Posiciones de Transformador, Línea, etc

- SOLINE: Líneas de Transmisión del SED

- SOTRAN: Transformadores del SED 
- SOLOAD: Cargas del SED

- SORTUS: RTUs utilizados en el sistema

- SOPROT: Protocolos utilizados en el sistema

- SOAREA: Nombre de las Áreas del SED

- SOZONA: Nombre de las Zonas del SED

Desde la figura 3.1 hasta la figura 3.15 se muestra capturas de pantalla con parte de las distintas tablas mencionadas, así mismo en el Anexo 1 se adjunta de manera digital el archivo accdb de Microsoft Access donde se encuentra la base de datos del sistema.

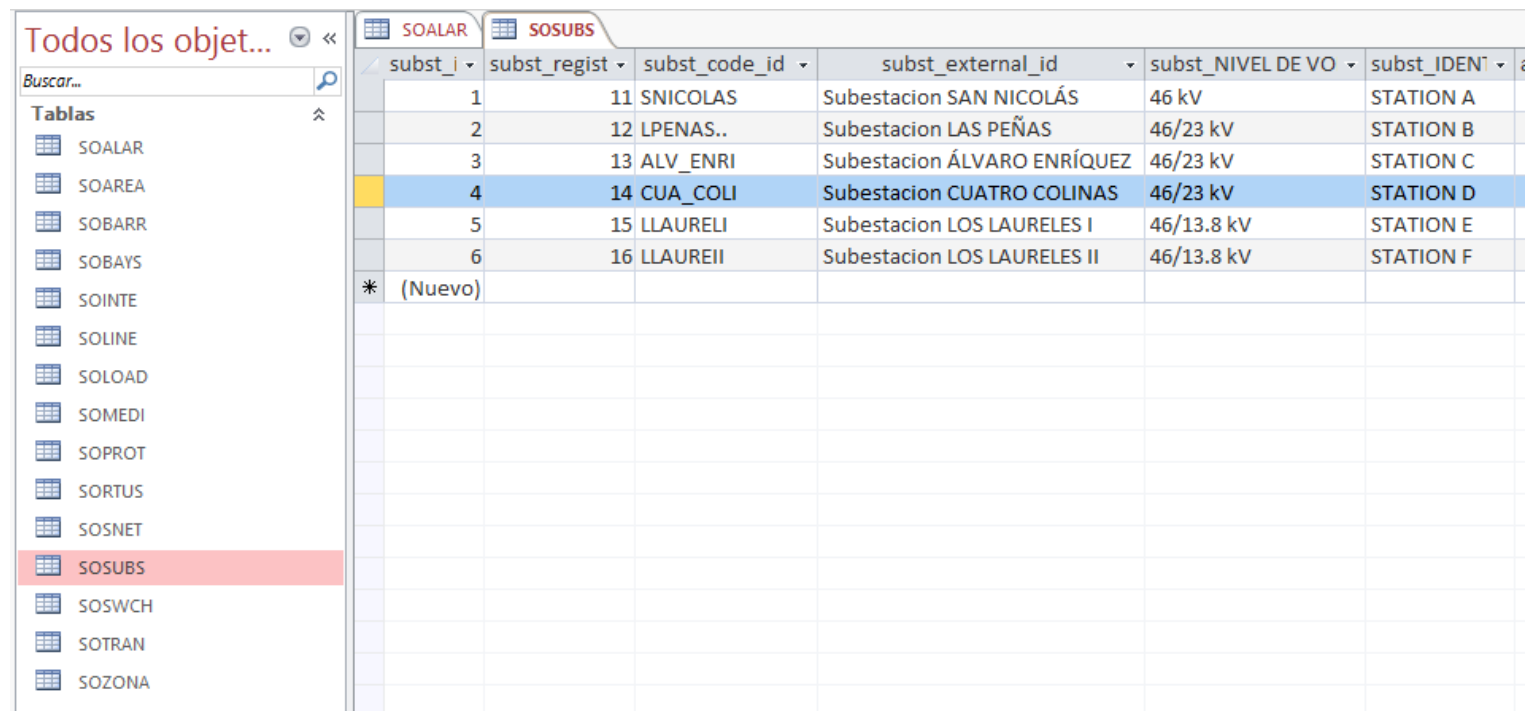

Figura 3. 1 Tabla SOSUBS

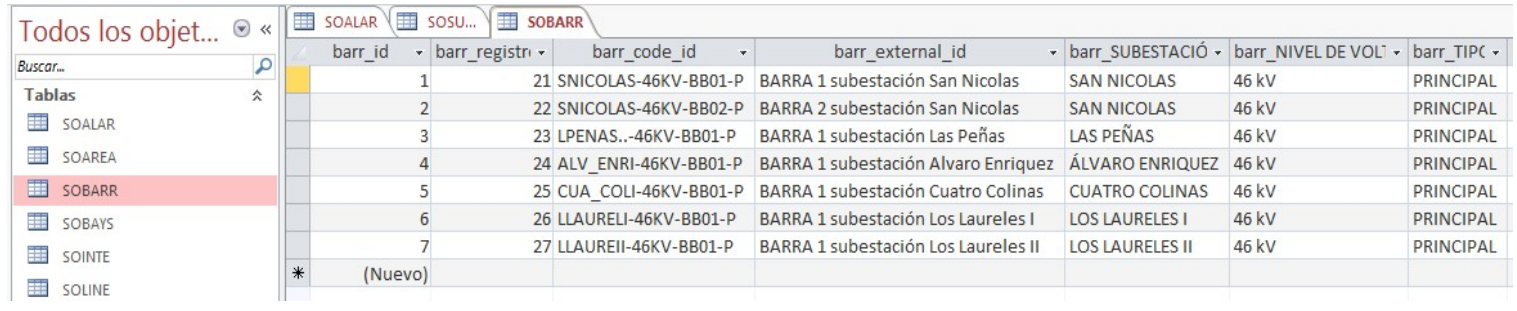

Figura 3. 2 Tabla SOBARR 
Guía de estilo para sistemas eléctricos de potencia

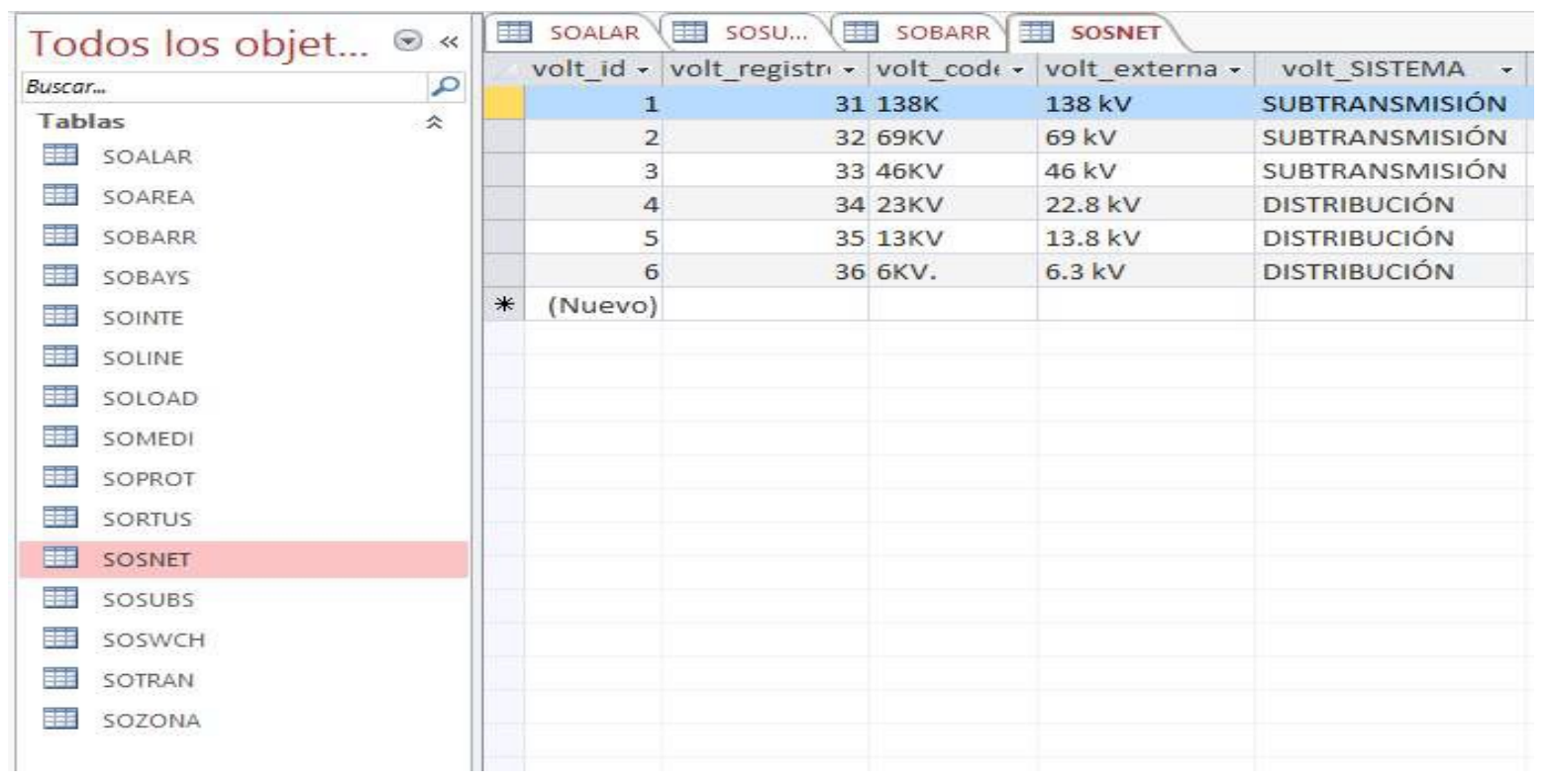

Figura 3. 3 Tabla SOSNET

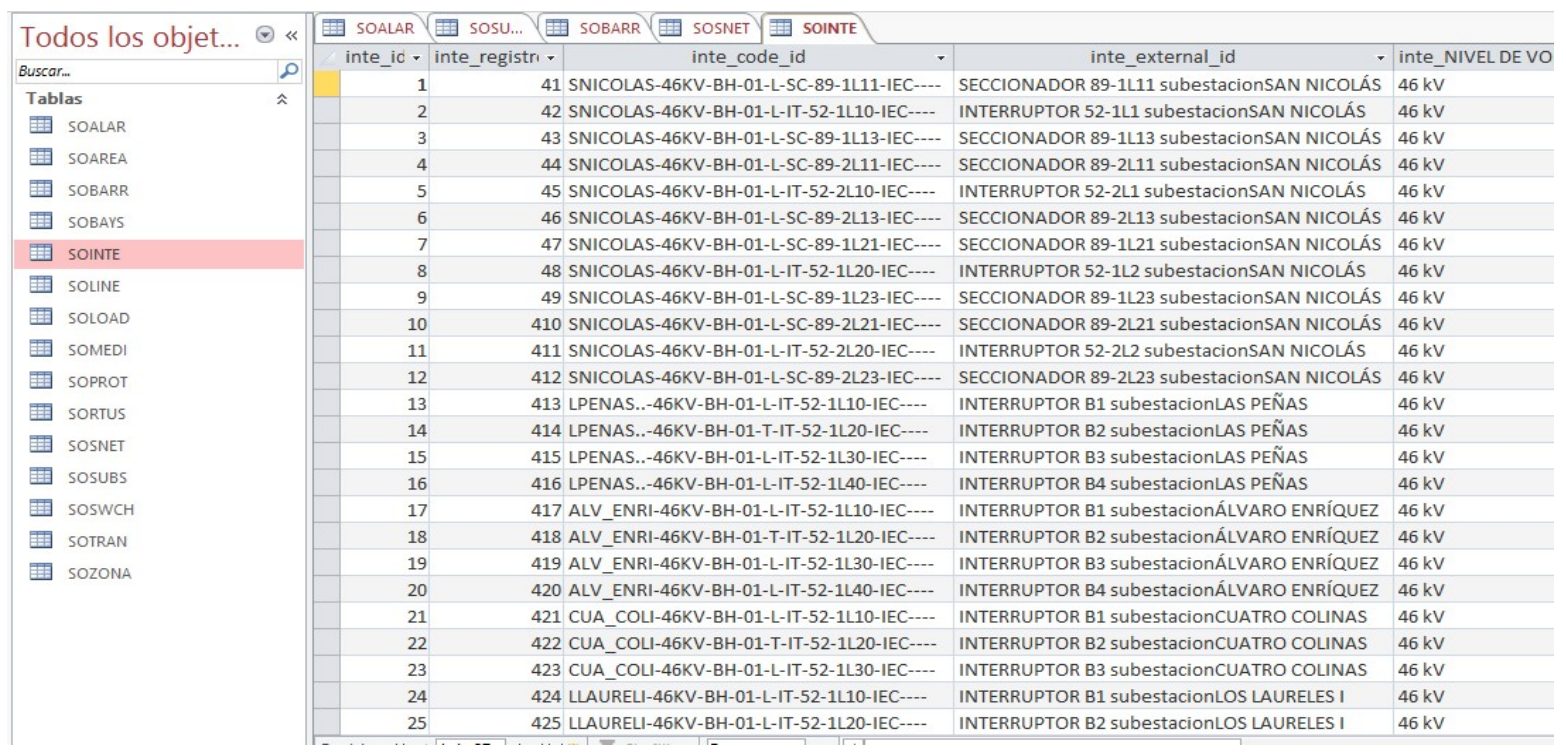

Figura 3. 4 Tabla SOINTE 


\begin{tabular}{|c|c|c|c|c|c|c|c|c|}
\hline J & & 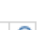 & \multicolumn{2}{|r|}{ swch_I. } & swch_regist . & swch_code_id & swch_external_id & swch_NIVEL_DE VI \\
\hline Buscar & & $\rho$ & & 1 & 51 & LPENAS..-46KV-BH-01-L-IT-52-1L10-IEC-..- & SWITCH B1 subestacion LAS PEÑAS & $46 \mathrm{kV}$ \\
\hline Tab & & $\hat{\imath}$ & & 2 & 52 & LPENAS..-46KV-BH-01-T-IT-52-1L20-IEC-.-. & SWITCH B2 subestacion LAS PEÑAS & $46 \mathrm{kV}$ \\
\hline & SOALAR & & & 3 & 53 & LPENAS..-46KV-BH-01-L-IT-52-1L30-IEC-..- & SWITCH B3 subestacion LAS PEÑAS & $46 \mathrm{kV}$ \\
\hline 困 & SOAREA & & & 4 & 54 & LPENAS..-46KV-BH-01-L-IT-52-1L40-IEC-..- & SWITCH B4 subestacion LAS PEÑAS & $46 \mathrm{kV}$ \\
\hline 困 & SOBARR & & & 5 & 55 & ALV_ENRI-46KV-BH-01-L-IT-52-1L10-IEC-.-- & SWITCH B1 subestacion ÁLVARO ENRÍQUEZ & $46 \mathrm{kV}$ \\
\hline 困 & SOBAYS & & & 6 & 56 & ALV_ENRI-46KV-BH-01-T-IT-52-1L20-IEC-..- & SWITCH B2 subestacion ÁLVARO ENRÍQUEZ & $46 \mathrm{kV}$ \\
\hline 圈 & SOINTE & & & 7 & 57 & ALV_ENRI-46KV-BH-01-L-IT-52-1L30-IEC-...- & SWITCH B3 subestacion ÁLVARO ENRÍQUEZ & $46 \mathrm{kV}$ \\
\hline & SOUNF & & & 8 & 58 & ALV_ENRI-46KV-BH-01-L-IT-52-1L40-IEC--.- & SWITCH B4 subestacion ÁLVARO ENRÍQUEZ & $46 \mathrm{kV}$ \\
\hline & SOLINE & & & 9 & 59 & CUA_COLI-46KV-BH-01-L-IT-52-1L10-IEC---- & SWITCH B1 subestacion CUATRO COLINAS & $46 \mathrm{kV}$ \\
\hline 围 & SOLOAD & & & 10 & 510 & CUA_COLI-46KV-BH-01-T-IT-52-1L20-IEC---- & SWITCH B2 subestacion CUATRO COLINAS & $46 \mathrm{kV}$ \\
\hline 雪 & SOMEDI & & & 11 & 511 & CUA_COLI-46KV-BH-01-L-IT-52-1L30-IEC---- & SWITCH B3 subestacion CUATRO COLINAS & $46 \mathrm{kV}$ \\
\hline 困 & SOPROT & & & 12 & 512 & LLAURELI-46KV-BH-01-L-IT-52-1L10-IEC---- & SWITCH B1 subestacion LOS LAURELES I & $46 \mathrm{kV}$ \\
\hline 困 & SORTUS & & & 13 & 513 & LLAURELI-46KV-BH-01-L-IT-52-1L20-IEC---- & SWITCH B2 subestacion LOS LAURELES I & $46 \mathrm{kV}$ \\
\hline & & & & 14 & 514 & LLAUREII-46KV-BH-01-L-IT-52-1L10-IEC--.. & SWITCH B1 subestacion LOS LAURELES II & $46 \mathrm{kV}$ \\
\hline 田 & SOSNET & & & 15 & 515 & LLAUREII-46KV-BH-01-L-IT-52-1L20-IEC--.. & SWITCH B2 subestacion LOS LAURELES II & $46 \mathrm{kV}$ \\
\hline 困 & SOSUBS & & * & (Nuevo) & & & & \\
\hline 团 & SOSWCH & & & & & & & \\
\hline
\end{tabular}

Figura 3. 5 Tabla SOSWCH

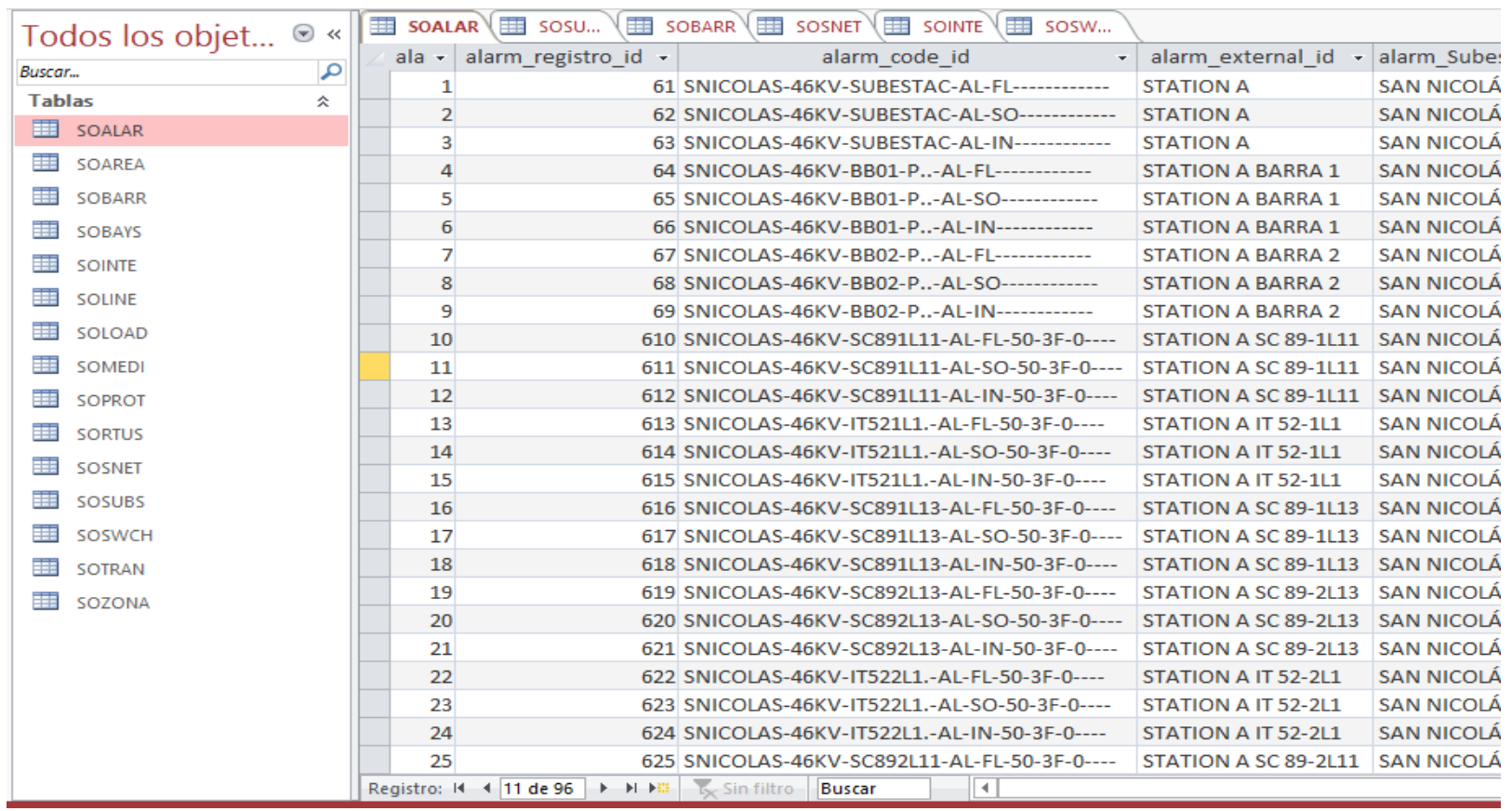

Figura 3. 6 Tabla SOALAR 


\begin{tabular}{|c|c|c|c|c|c|c|c|c|}
\hline \multirow{2}{*}{\multicolumn{3}{|c|}{ Todos los objet... $\odot \ll$}} & \multicolumn{3}{|c|}{ SOALAR SOSU... 囲 SOBARR } & \multicolumn{3}{|l|}{ SOMEDI } \\
\hline & & & me. & medi_regist - & medi_code_id & medi_external_id & medi_Subestació - & medi_NI \\
\hline \multicolumn{2}{|c|}{ Buscar... } & 2 & 1 & 71 & SNICOLAS-46KV-BB01-P..-MD-3F-0-V-KV--.-- & MEDIDA_VOLTAJE_BARRA1 & SAN NICOLAS & $46 \mathrm{kV}$ \\
\hline \multicolumn{2}{|c|}{ Tablas } & & 2 & 72 & SNICOLAS-46KV-BB02-P..-MD-3F-0-V-KV--.-- & MEDIDA_VOLTAJE_BARRA2 & SAN NICOLAS & $46 \mathrm{kV}$ \\
\hline \multicolumn{2}{|c|}{ SOALAR } & & 3 & 73 & SNICOLAS-46KV-BH-01...-MD-3F-0-V-KV-.--. & MEDIDA_VOLTAJE BAHIA1 & SAN NICOLAS & $46 \mathrm{kV}$ \\
\hline & SOAREA & & 4 & 74 & SNICOLAS-46KV-BH-02...-MD-3F-0-V-KV--.-- & MEDIDA VOLTAJE BAHIA2 & SAN NICOLAS & $46 \mathrm{kV}$ \\
\hline 困 & SOBARR & & 5 & 75 & LPENAS...-46KV-BB01-P..-MD-3F-0-V-KV-...- & MEDIDA VOLTAJE BARRAB & LAS PEÑAS & $46 \mathrm{kV}$ \\
\hline 困 & SOBAYS & & 6 & 76 & ALV-ENRI-46KV-BB01-P..-MD-3F-0-V-KV--..-- & MEDIDA VOLTAJE BARRAC & ALVARO ENRIQUEZ & $46 \mathrm{kV}$ \\
\hline \multirow{2}{*}{\multicolumn{2}{|c|}{ SOINTE }} & & 7 & 77 & CUA-COLI-46KV-BB01-P..-MD-3F-0-V-KV-..-- & MEDIDA VOLTAJE BARRAD & CUATRO COLINAS & $46 \mathrm{kV}$ \\
\hline & & & 8 & 78 & LLAURELI-46KV-BB01-P..-MD-3F-0-V-KV-.--- & MEDIDA VOLTAJE BARRAE & LOS LAURELES I & $46 \mathrm{kV}$ \\
\hline \multirow{2}{*}{\multicolumn{2}{|c|}{$\begin{array}{l}\text { 目 SOLINE } \\
\text { SOLOAD }\end{array}$}} & & 9 & 79 & LLAUREII-46KV-BB01-P..-MD-3F-0-V-KV--.-- & MEDIDA VOLTAJE BARRAF & LOS LAURELES II & $46 \mathrm{kV}$ \\
\hline & & & 10 & 710 & LPENAS..-46KV-IT-52-L1-MD-3F-0-V-KV-...- & MEDIDA VOLTAJE INT B1 SE_B & LAS PEÑAS & $46 \mathrm{kV}$ \\
\hline 团 & SOMEDI & & 11 & 711 & LPENAS..-46KV-IT-52-T1-MD-3F-0-V-KV-...- & MEDIDA VOLTAJE INT B2 SE_B & LAS PEÑAS & $46 \mathrm{kV}$ \\
\hline \multirow{2}{*}{\multicolumn{2}{|c|}{ SOPROT }} & & 12 & 712 & LPENAS..-46KV-IT-52-L2-MD-3F-0-V-KV-.--- & MEDIDA VOLTAJE INT B3 SE_B & LAS PEÑAS & $46 \mathrm{kV}$ \\
\hline & & & 13 & 713 & LPENAS...-46KV-IT-52-L3-MD-3F-0-V-KV-...- & MEDIDA VOLTAJE INT B4 SE_B & LAS PEÑAS & $46 \mathrm{kV}$ \\
\hline \multirow{2}{*}{\multicolumn{2}{|c|}{ SOSNET }} & & 14 & 714 & ALV-ENRI-46KV-IT-52-L1-MD-3F-0-V-KV-..-- & MEDIDA VOLTAJE INT B1 SE_C & ALVARO ENRIQUEZ & $46 \mathrm{kV}$ \\
\hline & & & 15 & 715 & ALV-ENRI-46KV-IT-52-T1-MD-3F-0-V-KV---- & MEDIDA VOLTAJE INT B2 SE_C & ALVARO ENRIQUEZ & $46 \mathrm{kV}$ \\
\hline \multicolumn{2}{|c|}{ sosubs } & & 16 & 716 & ALV-ENRI-46KV-IT-52-L2-MD-3F-0-V-KV-..- & MEDIDA VOLTAJE INT B3 SE_C & ALVARO ENRIQUEZ & $46 \mathrm{kV}$ \\
\hline \multirow{2}{*}{\multicolumn{2}{|c|}{$\begin{array}{l}\text { SOSWCH } \\
\text { SOTRAN }\end{array}$}} & & 17 & 717 & ALV-ENRI-46KV-IT-52-L3-MD-3F-0-V-KV-.--- & MEDIDA VOLTAJE INT B4 SE_C & ALVARO ENRIQUEZ & $46 \mathrm{kV}$ \\
\hline & & & 18 & 718 & CUA-COLI-46KV-IT-52-L1-MD-3F-0-V-KV--.-- & MEDIDA VOLTAJE INT B1 SE_D & CUATRO COLINAS & $46 \mathrm{kV}$ \\
\hline \multirow{8}{*}{\multicolumn{2}{|c|}{ SOZONA }} & & 19 & 719 & CUA-COLI-46KV-IT-52-T1-MD-3F-0-V-KV----- & MEDIDA VOLTAJE INT B2 SE_D & CUATRO COLINAS & $46 \mathrm{kV}$ \\
\hline & & & 20 & 720 & CUA-COLI-46KV-IT-52-L2-MD-3F-0-V-KV----- & MEDIDA VOLTAJE INT B3 SE_D & CUATRO COLINAS & $46 \mathrm{kV}$ \\
\hline & & & 21 & 721 & LLAURELI-46KV-IT-52-L1-MD-3F-0-V-KV----- & MEDIDA VOLTAJE INT B1 SE_E & LOS LAURELES I & $46 \mathrm{kV}$ \\
\hline & & & 22 & 722 & LLAURELI-46KV-IT-52-L2-MD-3F-0-V-KV----- & MEDIDA VOLTAJE INT B2 SE_E & LOS LAURELES I & $46 \mathrm{kV}$ \\
\hline & & & 23 & 723 & LLAUREII-46KV-IT-52-L1-MD-3F-0-V-KV--.-- & MEDIDA VOLTAJE INT B1 SE_F & LOS LAURELES II & $46 \mathrm{kV}$ \\
\hline & & & 24 & 724 & LLAUREII-46KV-IT-52-L2-MD-3F-0-V-KV-..-- & MEDIDA VOLTAJE INT B2 SE_F & LOS LAURELES II & $46 \mathrm{kV}$ \\
\hline & & & 25 & 725 & LPENAS..-23KV-TRAN-001-MD-3F-0-P-KW---.- & MEDIDA_POT_ACT_LOAD_T1_B & LAS PEÑAS & $23 \mathrm{kV}$ \\
\hline & & & Registro: 1 & 1 de 31 , & 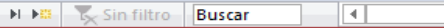 & & & \\
\hline
\end{tabular}

Figura 3. 7 Tabla SOMEDI

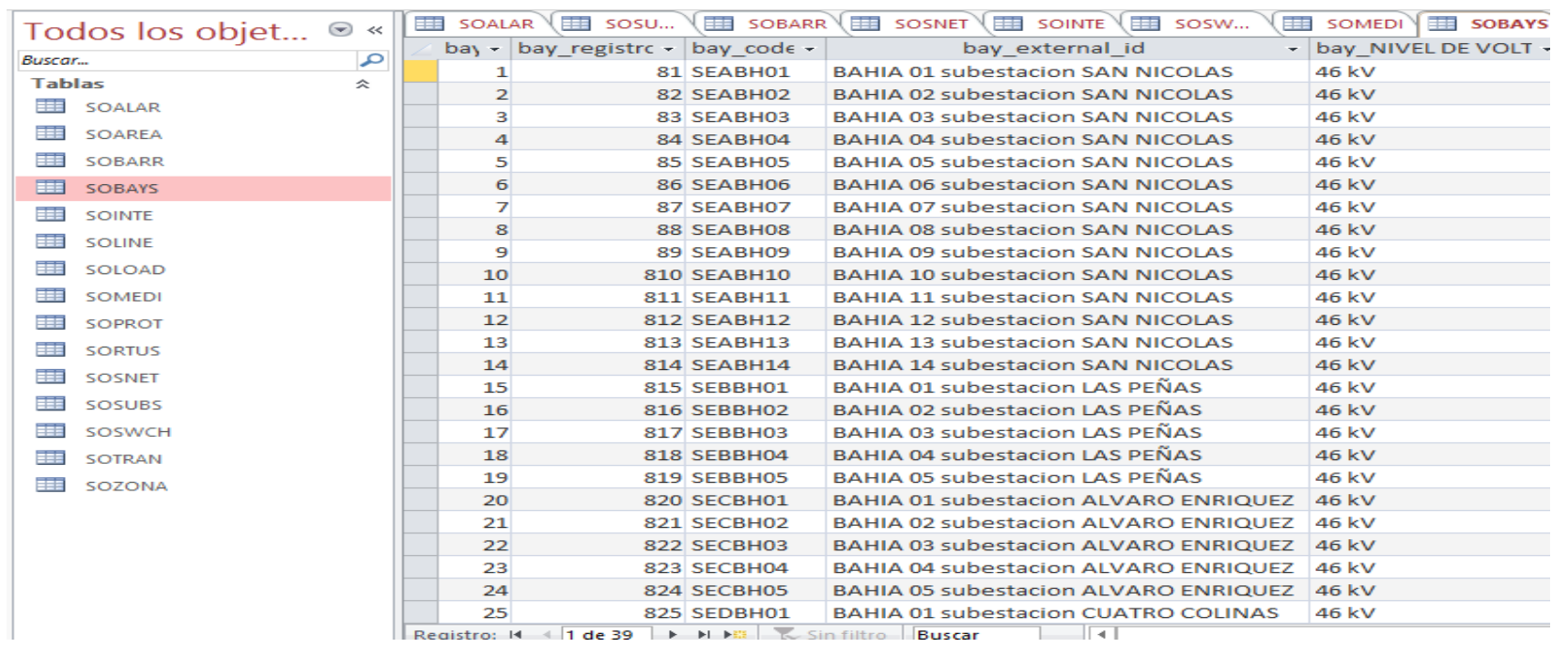

Figura 3. 8 Tabla SOBAYS 
Raul C. Ulloa De Souza; Alejandro J. Martinez-Peralta; Maria E. Moreno-Sanchez; Byron F. Chere-Quiñonez

\begin{tabular}{|c|c|c|c|c|c|c|c|}
\hline \multirow{3}{*}{\multicolumn{2}{|c|}{ Todos los objet... $\odot$ « }} & \multicolumn{2}{|l|}{ viuenal y IIItual } & Duscal & \multicolumn{3}{|c|}{ rommalo ue texiv } \\
\hline & & 团 SOALAR (国 SOSU... & 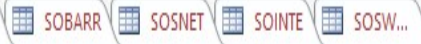 & SOMEDI SOBAYS & 团 SOLINE & & \\
\hline & & line__ll $\cdot$ line_registr & line_code_id & - line_Subestación de Sa . & line_Subestación de Llę̨ . & - line_NIVEL DEVVOL? & - line_Pote \\
\hline Buscar... & P & $-8-0$ & 91 SNICOLAS-LPENAS..-LTO1-CO1-46KV-100MVA.. & SAN NICOLAS & LAS PEÑAS & $46 \mathrm{kV}$ & $100 \mathrm{MVA}$ \\
\hline \multirow{2}{*}{$\begin{array}{l}\text { Tablas } \\
\text { 国 SOALAR }\end{array}$} & \multirow{6}{*}{$\hat{\imath}$} & 2 & 92 SNICOLAS-CUA_COLI-LTO1-CO1-46KV-100MVA.. & - SAN NICOLAS & CUATRO COLINAS & $46 \mathrm{kV}$ & $100 \mathrm{MVA}$ \\
\hline & & 3 & 93 LPENAS..-LLAURELI-LTO1-CO1-46KV-100MVA.. & LAS PEÑAS & LOS LAURELESI & $46 \mathrm{kV}$ & $100 \mathrm{MVA}$ \\
\hline 国 SOAREA & & 4 & 94 LLAURELI-LLAUREII-LTO1-CO1-46KV-100MVA.. & LOS LAURELESI & LOS LAURELES II & $46 \mathrm{kV}$ & $100 \mathrm{MVA}$ \\
\hline 国 SOBARR & & 5 & 95 CUA_COLL-ALV_ENRII-LT01-CO1-46KV-100MVA.. & - CUATRO COLINAS & ALVARO ENRIQUEZ & $46 \mathrm{kV}$ & $100 \mathrm{MVA}$ \\
\hline SOBAYS & & 6 & 96 ALV_ENRI-LLAUREII-LTO1-C01-46KV-100MVA... & ALVARO ENRIQUEZ & LOS LAURELES II & $46 \mathrm{kV}$ & $100 \mathrm{MVA}$ \\
\hline 用 SOINTF & & 7 & 97 LPENAS...-ALV_ENRI-LTO1-C01-46KV-100MVA.. & LAS PEÑNAS & ALVARO ENRIQUEZ & $46 \mathrm{kV}$ & 100 MVA \\
\hline
\end{tabular}

Figura 3. 9 Tabla SOLINE

\begin{tabular}{|c|c|c|c|c|c|c|c|}
\hline \\
\hline \multirow{2}{*}{\multicolumn{2}{|c|}{ Todos los objet... ๑ « }} & & SOALAR SOSU... & 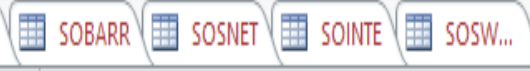 & SOMEDI SO & BAYS 四 SOLINE & IE $\#$ SOTRAN \\
\hline & \multirow{2}{*}{$\rho$} & & tran_ic - tran_registr & tran_code_id & - tran_Subestación. & tran_V prim. & tran_V secuno \\
\hline Buscar... & & & 11 & 101 LPENAS..-BH-01-T-TRAN-001-DY11-46KV-23KV & LAS PEÑAS & $46 \mathrm{kV}$ & $23 \mathrm{kV}$ \\
\hline \multirow{5}{*}{$\begin{array}{l}\text { Tablas } \\
\text { 围 SOALAR } \\
\text { 围 SOAREA } \\
\text { 囲 SOBARR } \\
\text { SOBAYS }\end{array}$} & \multirow{5}{*}{$\hat{\imath}$} & & 2 & 102 ALV_ENRI-BH-01-T-TRAN-001-DY11-46KV-23KV & ÁLVARO ENRÍQUEZ & $46 \mathrm{kV}$ & $23 \mathrm{kV}$ \\
\hline & & & 3 & 103 CUA_COLI-BH-01-T-TRAN-001-DY11-46KV-23KV & CUATRO COLINAS & $46 \mathrm{kV}$ & $23 \mathrm{kV}$ \\
\hline & & & 4 & 104 LLAURELI-BH-01-T-TRAN-001-DY11-46KV-13KV & LOS LAURELESI & $46 \mathrm{kV}$ & $13.8 \mathrm{kV}$ \\
\hline & & & 5 & 105 LLAUREII-BH-01-T-TRAN-001-DY11-46KV-113K & LOS LAURELES $\|$ & $46 \mathrm{kV}$ & $13.8 \mathrm{kV}$ \\
\hline & & * & (Nuevo) & & & & \\
\hline
\end{tabular}

Figura 3. 10 Tabla SOTRAN

\begin{tabular}{|c|c|c|c|c|c|c|c|c|}
\hline \multirow{2}{*}{\multicolumn{3}{|c|}{ Todos los objet... $\odot \ll$}} & \multicolumn{2}{|c|}{ SOALAR } & \multicolumn{4}{|c|}{ 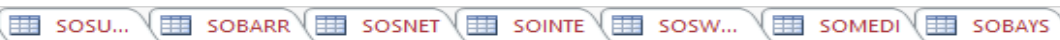 } \\
\hline & & & & load_ic - & load_registr . & load_code_id $\quad$. & load_Subestación - & load_NIVEL DE VOL' - \\
\hline \multicolumn{2}{|c|}{ Buscar... } & D & & 1 & 111 & LPENAS..-23KV-BH-01-T-L01 & LAS PEÑAS & $23 \mathrm{kV}$ \\
\hline \multicolumn{2}{|c|}{ Tablas } & \multirow[t]{5}{*}{$\hat{\imath}$} & & 2 & 112 & ALV_ENRI-23KV-BH-01-T-L01 & ÁLVARO ENRÍQUEZ & $23 \mathrm{kV}$ \\
\hline & SOALAR & & & 3 & 113 & CUA_COLI-23KV-BH-01-T-L01 & CUATRO COLINAS & $23 \mathrm{kV}$ \\
\hline 曾 & SOAREA & & & 4 & 114 & LLAURELI-13KV-BH-01-T-L01 & LOS LAURELES I & $13.8 \mathrm{kV}$ \\
\hline 击 & SOBARR & & & 5 & 115 & LLAUREII-13KV-BH-01-T-L01 & LOS LAURELES II & $13.8 \mathrm{kV}$ \\
\hline 围 & SOBAYS & & *⿻丷木 & (Nuevo) & & & & \\
\hline
\end{tabular}

Figura 3. 11 Tabla SOLOAD

Pol. Con. (Edición núm. 9) Vol. 2, No 8, agosto 2017, pp. 362-405, ISSN: 2550 - 682X 
Guía de estilo para sistemas eléctricos de potencia

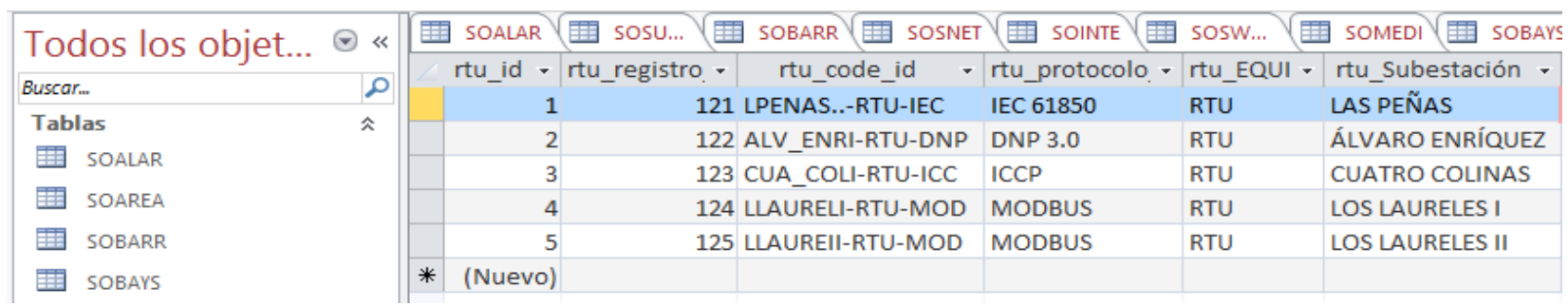

Figura 3. 12 Tabla SORTUS

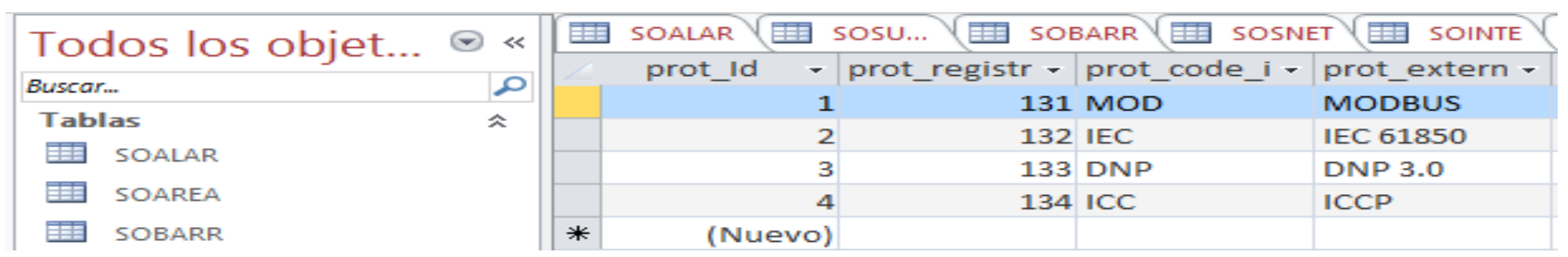

Figura 3. 13 Tabla SOPROT

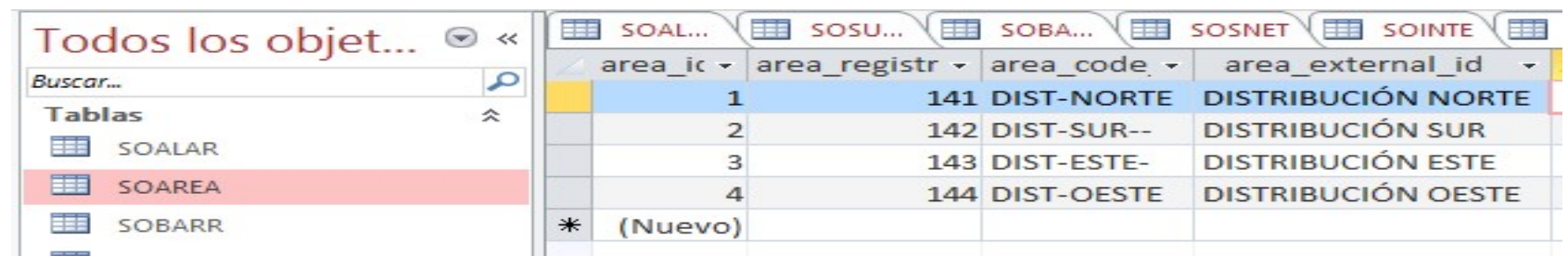

Figura 3. 14 Tabla SOAREA

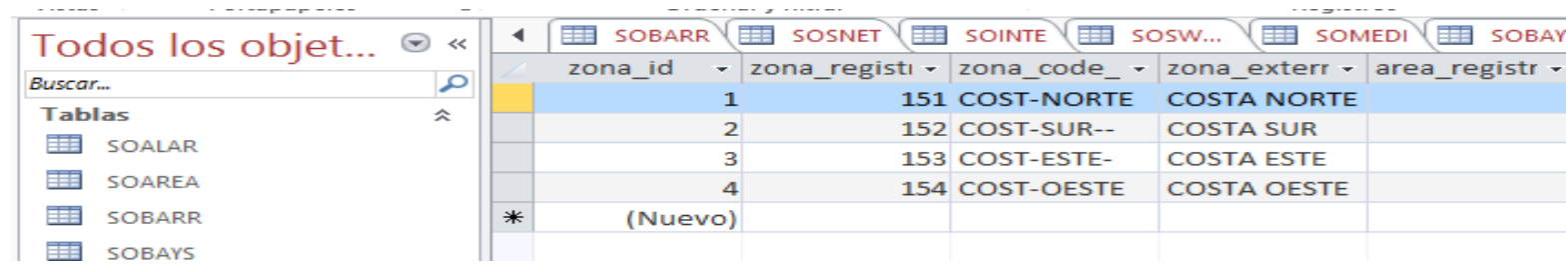

Figura 3. 15 Tabla SOZONA

Pol. Con. (Edición núm. 9) Vol. 2, No 8, agosto 2017, pp. 362-405, ISSN: 2550 - 682X 
Raul C. Ulloa De Souza; Alejandro J. Martinez-Peralta; Maria E. Moreno-Sanchez; Byron F. Chere-Quiñonez

\section{Interruptores y seccionadores}

Se establece un documento .xls mediante el software Microsoft Excel llamado "Interruptores y Seccionadores" en el cual se determina el estado inicial de interruptores y seccionadores del sistema, para esto se utiliza código binario en el cual se establece que la condición "1" significa que el elemento se encuentra en estado de conexión y a su vez que la condición " 0 ” nos indica que el elemento se encuentra abierto. En la figura 3.16 se muestra una captura de pantalla donde se muestra el archivo mencionado, así mismo en el Anexo 2 se adjunta de manera digital el archivo .xls de Microsoft Excel donde se encuentra el estado inicial de los interruptores y seccionadores del sistema.

\begin{tabular}{|c|c|c|c|c|c|c|}
\hline 1 & \multicolumn{6}{|c|}{ INTERRUPTORES Y SECCIONADORES } \\
\hline 2 & Subestación & ificacion subest & Identificación de Campo & Identidad Externa & Identidad Unica & Estado Inicial \\
\hline 3 & SAN NICOLÁS & STATION-A & \begin{tabular}{|r|}
$89-1 \mathrm{~L} 11$ \\
\end{tabular} & 89-1L11 & 401 & 1 \\
\hline 4 & SAN NICOLÁS & STATION-A & 52-1L1 & $52-1 \mathrm{~L} 1$ & 402 & 1 \\
\hline 5 & SAN NICOLÁS & STATION-A & 89-1L13 & 89-1L13 & 403 & 1 \\
\hline 6 & SAN NICOLÁS & STATION-A & 89-2L11 & 89-2L11 & 404 & $\theta$ \\
\hline 7 & SAN NICOLÁS & STATION-A & $52-2 \mathrm{~L} 1$ & $52-2 \mathrm{~L} 1$ & 405 & 0 \\
\hline 8 & SAN NICOLÁS & STATION-A & 89-2L13 & 89-2L13 & 406 & 0 \\
\hline 9 & SAN NICOLÁS & STATION-A & 89-1L21 & 89-1L21 & 407 & 0 \\
\hline 10 & SAN NICOLÁS & STATION-A & $52-1 \mathrm{~L} 2$ & $52-1 \mathrm{~L} 2$ & 408 & 0 \\
\hline 11 & SAN NICOLÁS & STATION-A & 89-1L23 & 89-1L23 & 409 & 0 \\
\hline 12 & SAN NICOLÁS & STATION-A & $89-2 L 21$ & $89-2 \mathrm{~L} 21$ & 410 & 1 \\
\hline 13 & SAN NICOLÁS & STATION-A & $52-2 \mathrm{~L} 2$ & $52-2 \mathrm{~L} 2$ & 411 & 1 \\
\hline 14 & SAN NICOLÁS & STATION-A & $89-2 L 23$ & $89-2 L 23$ & 412 & 1 \\
\hline 15 & LAS PEÑAS & STATION-B & B1 & $52-1 \mathrm{~L} 1$ & 413 & 1 \\
\hline 16 & LAS PEÑAS & STATION-B & B2 & $52-1 \mathrm{~T} 1$ & 414 & 1 \\
\hline 17 & LAS PEÑAS & STATION-B & B3 & $52-1 L 2$ & 415 & 1 \\
\hline 18 & LAS PEÑAS & STATION-B & B4 & $52-1 L 3$ & 416 & 1 \\
\hline 19 & ÁLVARO ENRÍQUEZ & STATION-C & B1 & $52-1 \mathrm{~L} 1$ & 417 & 1 \\
\hline 20 & ÁLVARO ENRÍQUEZ & STATION-C & B2 & $52-1 \mathrm{~T} 1$ & 418 & 1 \\
\hline 21 & ÁLVARO ENRÍQUEZ & STATION-C & B3 & $52-1 L 2$ & 419 & 1 \\
\hline 22 & ÁLVARO ENRÍQUEZ & STATION-C & B4 & $52-1 L 3$ & 420 & 1 \\
\hline 23 & CUATRO COLINAS & STATION-D & B1 & 52-1L1 & 421 & 1 \\
\hline 24 & CINATON CNI TAIAC & CTATTOM $n$ & on & Er $1 \mathrm{~T}_{1}$ & 127 & 1 \\
\hline & Estado & † & & & & $\vdots$ \\
\hline
\end{tabular}

Figura 3. 16 Estado inicial de Interruptores y Seccionadores 


\section{Código Principal: "Topología del Sistema"}

Es el código principal, el cual ha sido realizado mediante el uso de la base de datos desarrollada en Microsoft Access mencionada en el inciso $3.1 \mathrm{y}$ un algoritmo programado en Matlab, debido a que el proyecto busca informar a un determinado usuario acerca del estado de conexión de equipos mediante la evaluación de apertura o cerrado de interruptores, se ha utilizado lógica booleana ya que permite establecer dos valores únicamente lo cual concuerda con la lógica del funcionamiento de interruptores mecánicos. En el Anexo 3 se adjunta de manera digital la carpeta donde se encuentra el archivo .m con el código o programa principal para ser utilizado en el software Matlab; así mismo se presenta los archivos .m correspondientes a las diferentes funciones a las que se le hace llamado desde el código principal, tal es el caso la función para cargar tabla de base de datos, función para cargar el estado inicial de interruptores y seccionadores; y las funciones para evaluar transformadores, líneas e interruptores.

$\mathrm{Al}$ inicio se debe leer las tablas que componen la base de datos, y el estado inicial de los interruptores que se deberá detallar en el documento de Excel mencionado en el inciso anterior, posteriormente se procede con la evaluación de los interruptores que conectan las Barras 1 y 2 de la Estación A, si dichas líneas permiten una conexión con las barras B o D se asume que se puede tener un SISTEMA OPERATIVO caso contrario se considera un SISTEMA NO OPERATIVO y el programa culminaría. En la figura 3.17 se muestra el algoritmo utilizado. 


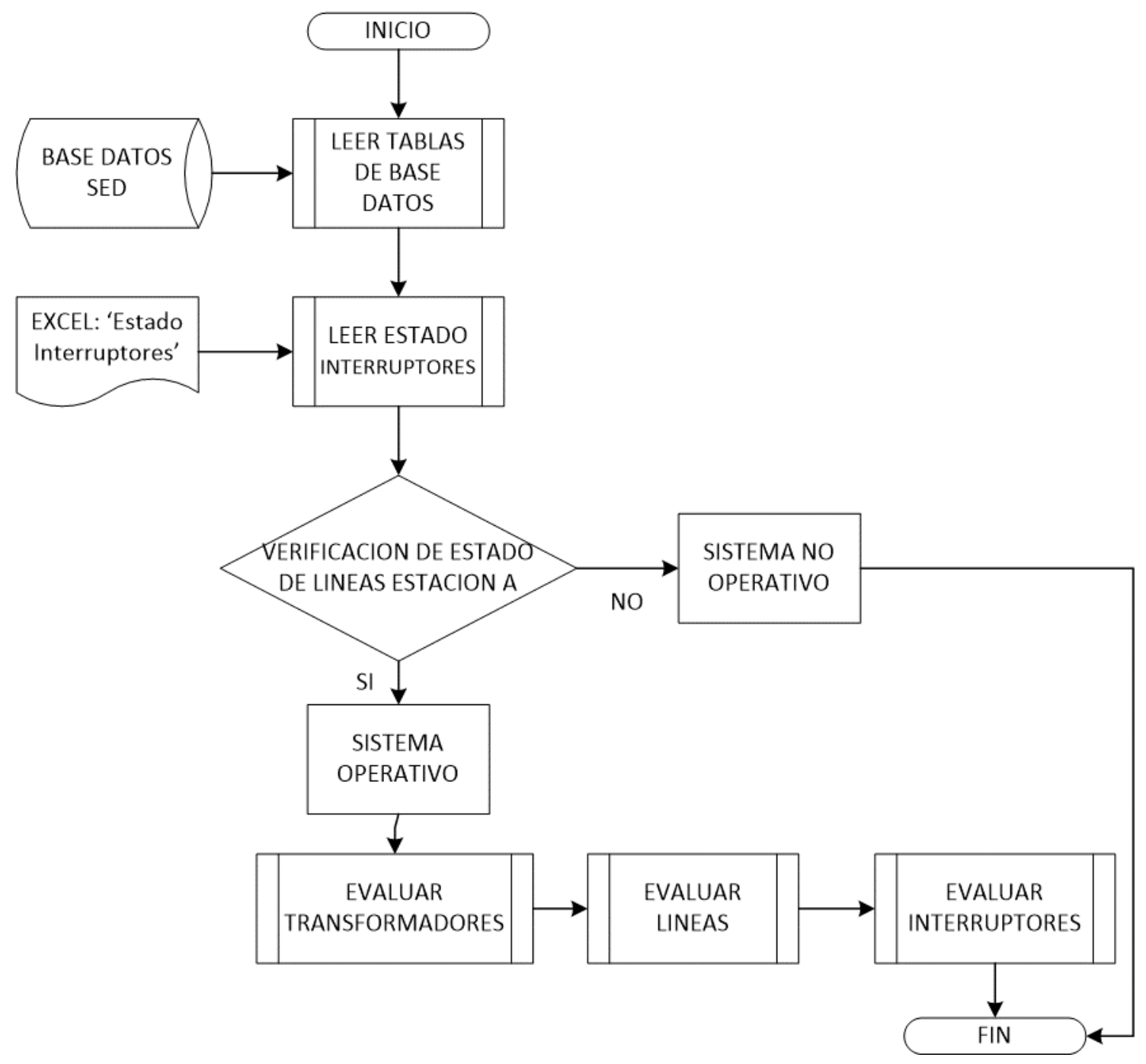

Figura 3. 17 Algoritmo utilizado

Para un correcto uso del código de Matlab propuesto en el presente Proyecto Técnico es necesario establecer las siguientes instrucciones:

- El código se halla compuesto por varios archivos de Matlab, la base de datos creada en ACCESS y el archivo Excel donde se encuentra el estado de los interruptores.

- Matlab debe ser capaz de leer la base de datos generada en ACCESS, para lo cual es necesario que cuente con el complemento "Database Toolbox" 
- En el momento de iniciar el programa se puede optar por dos alternativas: la una implica ejecutar el código CargarTablas, en la línea 8 se cambia la ubicación de la base de datos y la otra es que en la línea 8 del programa CodigoTopologiaSED se aplaste simultáneamente ctrl $+\mathrm{D}$, que abre la función CargaTablas y se procede a cambiar/actualizar la ubicación de la base de datos.

- Una vez cambiada para evaluar la topología se cambiará LOS ESTADOS DE LOS INTERRUPTORES EN EL ARCHIVO EXCEL. Después de cada cambio hay que grabar previo a la ejecución del script del programa principal.

En los incisos subsiguientes se explica las funciones para evaluar transformadores, líneas e interruptores.

\section{Función: "Evaluación de Transformadores"}

Para esta evaluación se toma en cuenta todas las combinaciones posibles de los interruptores que deben estar conectados desde la estación A hasta el transformador, si dichos interruptores tienen un estado inicial de ' 1 ' en todo el recorrido posible se considera que el transformador está EN SERVICIO, en caso de que alguno de estos interruptores en alguna de las combinaciones posibles posee un estado de ' 0 ' se desecha dicha combinación y si de ser el caso si todas las combinaciones posibles dieran como resultado '0' se considera al transformador como FUERA DE SERVICIO.

\section{Función: "Evaluación de Líneas"}

Previo a la evaluación de las líneas se realiza un diagnóstico de las líneas independientemente, para fijar un valor lógico a todas las líneas que conforman el sistema, en este procedimiento si los dos extremos de una línea conformados por un interruptor se encuentran 
conectados se considera a la línea con un estado lógico de ' 1 ', caso contrario si los dos o alguno de los extremos de la línea se encuentra desconectada y se le asigna un valor lógico de ' 0 '.

Para la presente función se evalúa el funcionamiento de las líneas que se encuentran antes o después de alguno de los extremos de la línea en diagnóstico, es decir para que una línea se considere EN SERVICIO todas las líneas que permiten la conexión desde la Estación A hasta la línea evaluada deberán presentar un estado lógico de ' 1 ', en el caso que alguna de ellas posea un valor de ' 0 ' se desecha dicha combinación, de igual manera que en la evaluación de transformadores si todas las posibles combinaciones dieran como resultado un ' 0 ' se considera a la línea como FUERA DE SERVICIO.

Para finalizar con este proceso se evalúa los extremos de cada línea con la finalidad de conocer el estado de conexión de los interruptores que componen la línea, si el interruptor posee un estado inicial de ' 1 ' se mostrara EN SERVICO, caso contrario si fuera un '0' se mostrara FUERA DE SERVICIO.

\section{Función: "Evaluación de Interruptores"}

Para la evaluación de los diferentes interruptores, se toma en cuenta el estado inicial que se asignó en el documento de Excel a cada uno de los equipos, si el estado lógico de determinado equipo es '0’ se mostrará un mensaje de FUERA DE SERVICIO caso contrario si el valor lógico fuera de '1' se mostrará un mensaje de EN SERVICIO.

Para cada una de las evaluaciones se trabaja con el código de identificación que se encuentra en cada una de las tablas, así se puede buscar de una mejor manera el índice al cual pertenece dicho elemento dentro de la tabla obtenida desde la base de datos. 


\section{Resultados del Código Principal: "Topología del Sistema”}

En el presente inciso se muestran los resultados obtenidos al correr el código “Topología del Sistema" en donde se detalla el sistema operativo del SEP analizado, considerando el estado de operación de los transformadores, líneas de transmisión; interruptores y seccionadores.

En las figuras $3.18,3.19$ y 3.20 se muestran las capturas de pantalla de los resultados obtenidos en el Matlab.

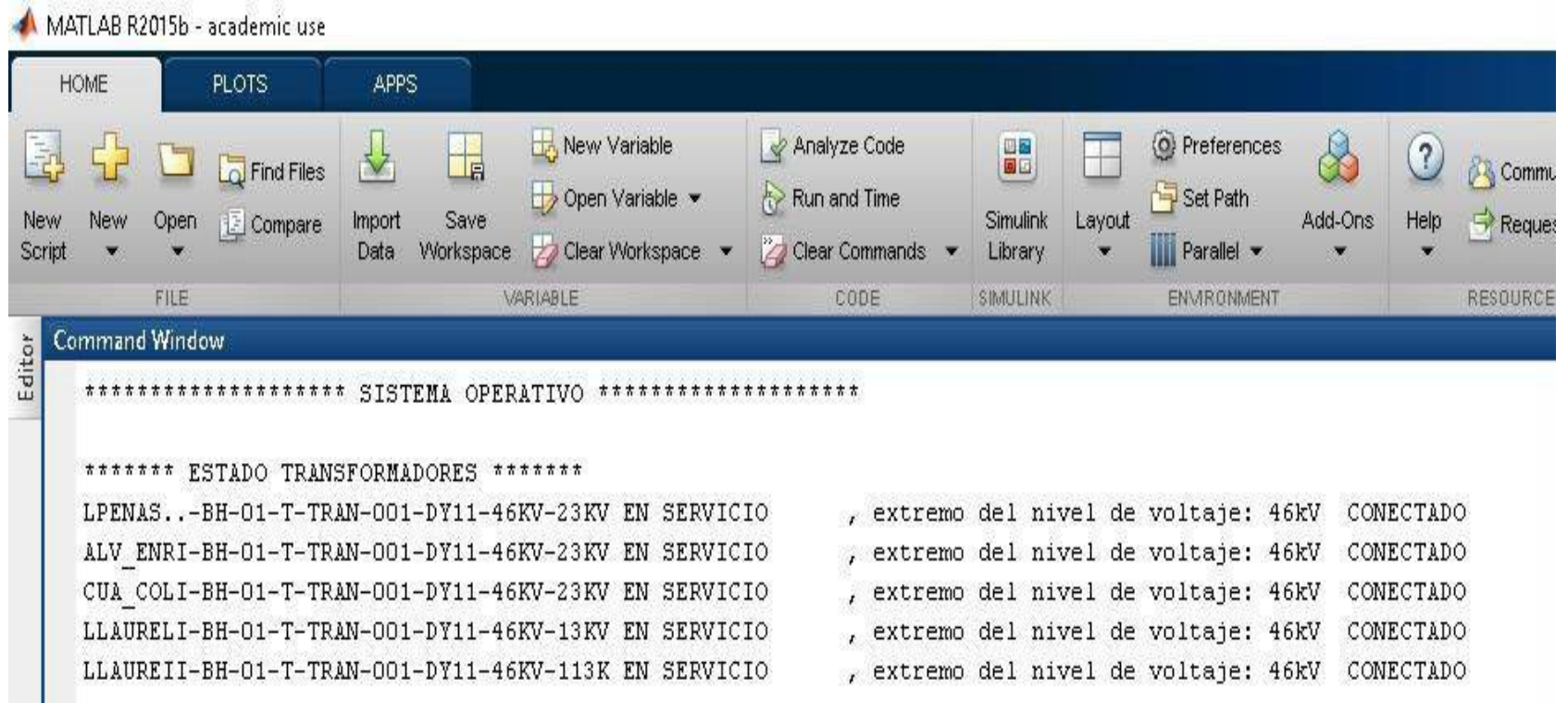

Figura 3. 18 Resultados: Estado de los transformadores 
Raul C. Ulloa De Souza; Alejandro J. Martinez-Peralta; Maria E. Moreno-Sanchez; Byron F. Chere-Quiñonez

ATLAB R2015b - academicuse

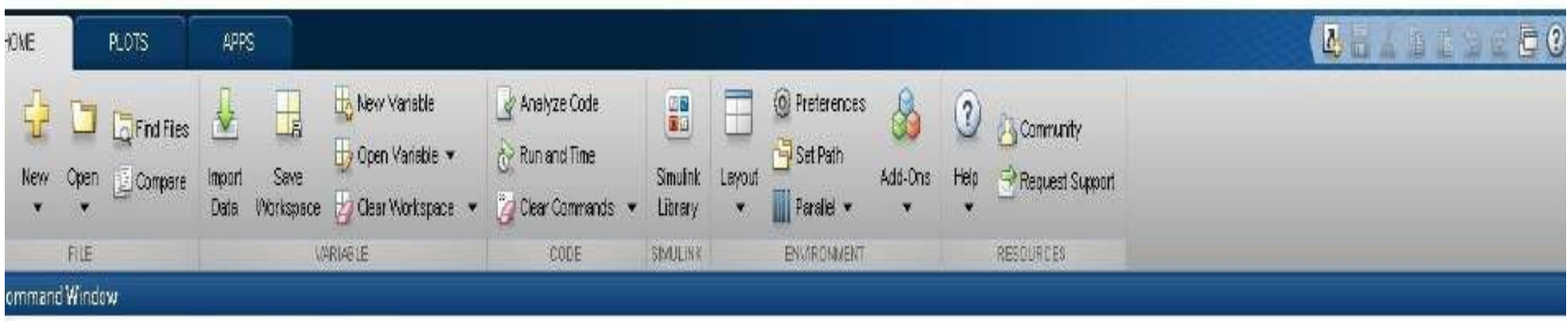

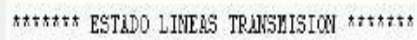

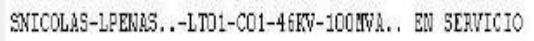

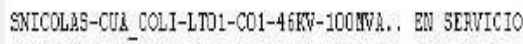

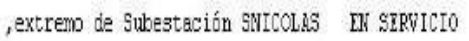

,excremo de Subestación SNICOLls Dis SIRVICI0

,extrem de Subestación LPENiS.. DI SIRVICIO

0 , extremo de Subestación LLAURELI

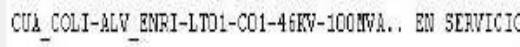

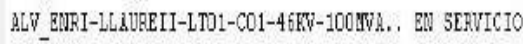

LPENLS..-ALV _ENRI-LTO1-CO1-46KN-100W\% . EN SERVICIO ,extremo de Subestación COL COLI DIS SZRVICIO

,excremo de Subestación RLV EURI EN SIRVICIO

,extrem de Subestación LPENiS. D DISRVICIO
, extremo de Subestacion LeENDS.. EN SERvicio

- extremo de Subestacion COS COLI EN SERVICIO

, extremo de Subestacion LLiJRELI EN SERVICIO

, extremo de Subestacion LLdUREII FURR DE SERVICTO

- extremo de Subestacion ALV INRI EN SERVICIO

- extremo de Subestacion LLdUdeII EN SERWTCIO

- extremo de Subestacion ALV INRI EN SERICIO

Figura 3. 19 Resultados: Estado de Líneas de Transmisión 
MATLAB R2015b - academic use

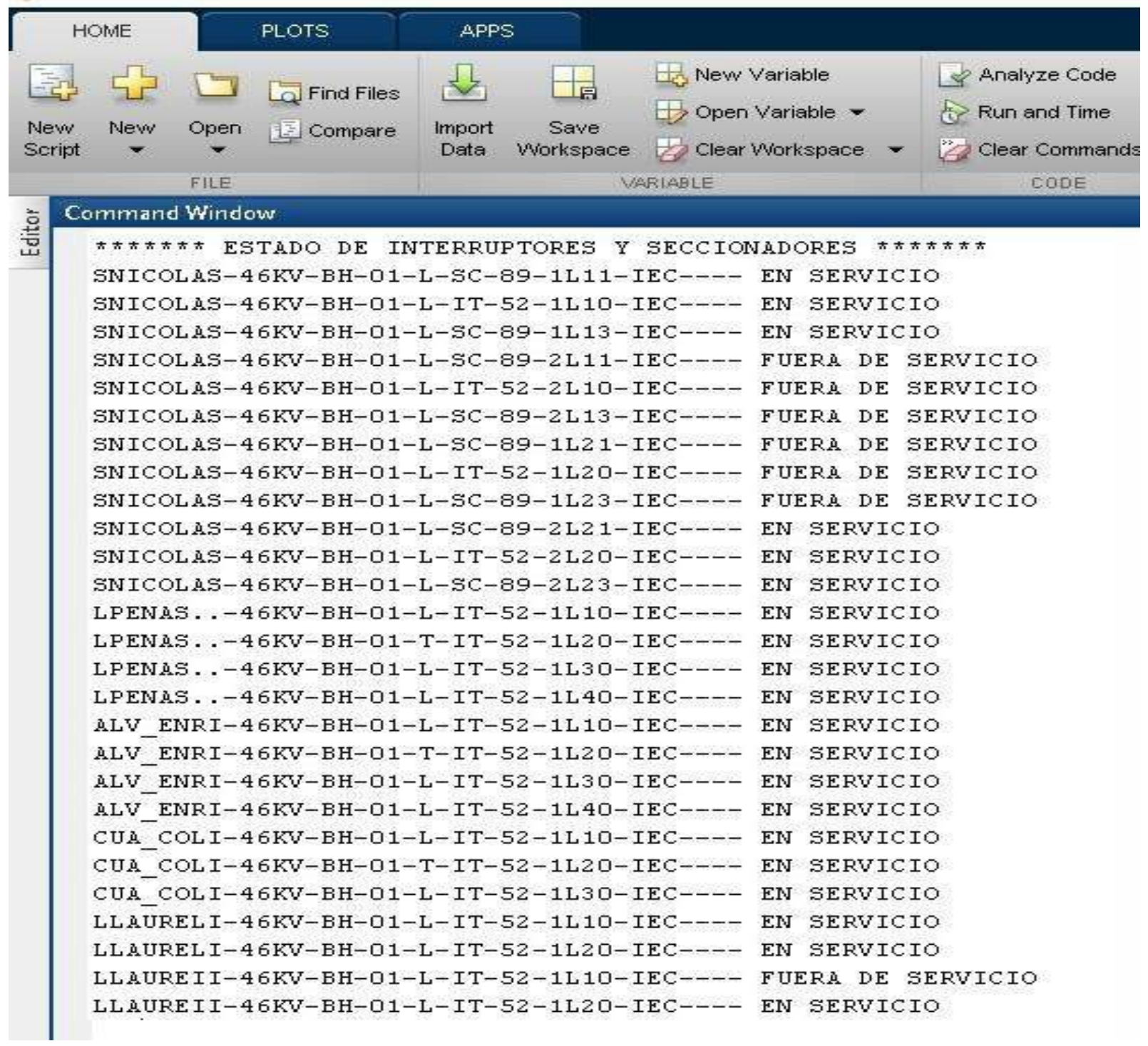

Figura 3. 20 Resultados: Estado de Interruptores y Seccionadores

Como se puede observar en las figuras adjuntas se muestra los resultados obtenidos del código de programación propuesto, en la figura 3.18 se tiene el estado de los transformadores, donde todos se encuentran en servicio, así mismo se detalla la conexión del lado de $46 \mathrm{kV}$.

En la figura 3.19 se detalla el estado de conexión de las líneas de transmisión, donde se puede observar que cada una de ellas se encuentra en estado de servicio a excepción de la línea "Los 
Laureles I" la misma que se encuentra desconectada en el extremo que se une a la subestación "Los Laureles”. Así mismo se describe la subestación a la cual se enlaza cada uno de los extremos de cada línea de transmisión analizada y el estado de conexión de los mismos.

Para culminar el análisis de los resultados del presente proyecto se muestra la figura 3.20 donde se detalla el estado de conexión de los interruptores y seccionadores, de lo que se puede observar que veinte se encuentran en servicio y siete en estado de desconexión.

\section{Conclusiones}

El conocimiento sobre el manejo de las bases de datos y de bases de relacionales se hace imprescindible para entender y organizar la infinidad de datos que se pueden obtener tanto dentro de los sistemas eléctricos de potencia como los de distribución, lo cual resulta conveniente para poder establecer un Modelo de Información Común CIM que permita la integración y el intercambio de información entre las distintas aplicaciones de los sistemas de información para el manejo de los SSEE.

La posibilidad de manejar información procedente de varias fuentes en una sola plataforma permite la interoperatividad de los sistemas y de los equipos. En el presente proyecto se pudo visualizar que el programa Matlab permite leer archivos Excel y archivos ACCESS lo que facilita la ejecución del programa.

La guía de estilo es muy importante y permite definir un mismo nivel de lenguaje que se debe manejar dentro de los sistemas de información para manejo y gestión de los SSEE. 
Es imprescindible conocer las relaciones efectivas y reales que se dan entre las diferentes tablas de una base de datos de los sistemas de información para poder relacionarlas adecuadamente, dándole relevancia e importancia a una correcta guía de estilo de los elementos del SSEE.

La nomenclatura homologada de los elementos que comprende un SSEE permite una correcta identificación de cada uno de estos elementos dentro de los sistemas de información utilizados para manejo y gestión de los SSEE.

El código de programación desarrollado en el presente se encuentra encriptado de forma general, de tal manera que puede ser utilizado para cualquier SSEE cuyos elementos hayan sido ingresados previamente en una base de datos siguiendo la guía de estilo detallada.

\section{Bibliografia.}

[1] S. Ramírez, "Redes de Distribución de Energía", Universidad Nacional de Colombia, Tercera Edición, Manizales, 2012.

[2] A. Vargas, "Sistemas de Información en Empresas Distribuidoras", Curso de Posgrado, Universidad Politécnica Nacional, Quito, 2016.

[3] L. Tapia, “Operación de Subestaciones”, Escuela Politécnica Nacional, Quito, Ecuador, 2005.

[4] M. Granada, "Estimación de Estado en Sistemas Eléctricos de Potencia: Parte I Detección de Errores Grandes”, Universidad Tecnológica de Pereira, Colombia, 2003.

[5] H. Edelmann, “A Universal Assessment For The Superior Quality Of Distribution Of Measuring Points For The State Estimation Of High Voltage Networks", Proceedings Power System Computation Conference, Paper 2.3/7, September 1975.

[6] P. Zarco, A. Gómez, "Estimación de Estado y Parámetros en Redes Eléctricas”, Departamento de Ingeniería Eléctrica de la Universidad de Sevilla, España, 1999.

[7] A. Rodríguez Penin, "Sistema de Visualización Industrial,” Marcombo, Pp. 3-91, 2006.

[8] R. Santo Domingo, J. Rodríguez, And E. Pilo de la Fuente, "Introducción Al Modelo Cim de los Sistemas de Energía".

[9] L. King, “The Common Information Model For Distribution,” Management, Pp. 1-3, 2008.

[10] O. Huisman And R. A De By, Principles Of Geographic Information Systems An Introductory Texbook. 2009. 
Raul C. Ulloa De Souza; Alejandro J. Martinez-Peralta; Maria E. Moreno-Sanchez; Byron F. Chere-Quiñonez

[11] Sustainable Energy Regulation And Policymaking For Africa, "Module 15 Demand-Side Management Advance Copy". 